\title{
MUSIELAK-ORLICZ HARDY SPACES ASSOCIATED WITH DIVERGENCE FORM ELLIPTIC OPERATORS WITHOUT WEIGHT ASSUMPTIONS
}

\author{
TRI DUNG TRAN
}

\begin{abstract}
Let $L$ be a divergence form elliptic operator with complex bounded measurable coefficients, let $\omega$ be a positive Musielak-Orlicz function on $(0, \infty)$ of uniformly strictly critical lower-type $p_{\omega} \in(0,1]$, and let $\rho(x, t)=$ $t^{-1} / \omega^{-1}\left(x, t^{-1}\right)$ for $x \in \mathbb{R}^{n}, t \in(0, \infty)$. In this paper, we study the MusielakOrlicz Hardy space $H_{\omega, L}\left(\mathbb{R}^{n}\right)$ and its dual space $\mathrm{BMO}_{\rho, L^{*}}\left(\mathbb{R}^{n}\right)$, where $L^{*}$ denotes the adjoint operator of $L$ in $L^{2}\left(\mathbb{R}^{n}\right)$. The $\rho$-Carleson measure characterization and the John-Nirenberg inequality for the space $\mathrm{BMO}_{\rho, L}\left(\mathbb{R}^{n}\right)$ are also established. Finally, as applications, we show that the Riesz transform $\nabla L^{-1 / 2}$ and the Littlewood-Paley $g$-function $g_{L}$ map $H_{\omega, L}\left(\mathbb{R}^{n}\right)$ continuously into $L(\omega)$.
\end{abstract}

\section{$\S 1$. Introduction}

The introduction and study of classical real-variable Hardy and BMO spaces on the Euclidean space $\mathbb{R}^{n}$ began in the 1960 s with an initial article of Stein and Weiss [32]. Later, this theory was developed systematically by Fefferman and Stein [18] and studied extensively in [13] and [31] as well by many others. Since then these classes of functions have played an important role in a number of analyses, such as modern harmonic analysis and partial differential equations. It is now well known that there are various equivalent characterizations of functions in the classical Hardy space. For instance, the Hardy space $H^{1}\left(\mathbb{R}^{n}\right)$ can be viewed as the set of functions $f \in L^{1}\left(\mathbb{R}^{n}\right)$ such that the Riesz transform $\nabla(\Delta)^{-1 / 2} f$ belongs to $L^{1}\left(\mathbb{R}^{n}\right)$. We also have alternative characterizations of $H^{1}\left(\mathbb{R}^{n}\right)$ via the atomic decomposition or by the square function and the nontangential maximal function associated to the Poisson semigroup generated by the Laplacian. Basically, this standard

Received November 8, 2012. Revised April 27, 2013. Accepted October 12, 2013.

First published online October 7, 2014.

2010 Mathematics Subject Classification. Primary 42B20; Secondary 35B65, 35K05, 42B25, 47B38, 58J35. 
theory of Hardy spaces is intimately connected with properties of harmonic functions and of the Laplacian.

Nevertheless, it is a fact that there are some situations in which the classical Hardy spaces are not applicable. For example, one considers a general elliptic operator in divergence form with complex bounded coefficients. Let $A$ be an $(n \times n)$-matrix with entries $\left\{a_{j, k}\right\}_{j, k=1}^{n} \subset L^{\infty}\left(\mathbb{R}^{n}, \mathbb{C}\right)$ satisfying the ellipticity conditions; namely, there exist constants $0<\lambda_{A} \leq \Lambda_{A}<\infty$ such that for all $\xi, \zeta \in \mathbb{C}^{n}$,

$$
\lambda_{A}|\xi|^{2} \leq \mathcal{R} e\langle A \xi, \xi\rangle \quad \text { and } \quad|\langle A \xi, \zeta\rangle| \leq \Lambda_{A}|\xi||\zeta|
$$

Then the second-order divergence form operator is given by

$$
L f \equiv-\operatorname{div}(A \nabla f)
$$

interpreted in the weak sense via a sesquilinear form.

It is shown that the Riesz transform $\nabla L^{-1 / 2}$ is bounded on $L^{2}\left(\mathbb{R}^{n}\right)$ but not bounded from $H^{1}\left(\mathbb{R}^{n}\right)$ to $L^{1}\left(\mathbb{R}^{n}\right)$ (for more details, see [22]). The need for research of new Hardy spaces other than the Hardy space $H^{1}\left(\mathbb{R}^{n}\right)$ thus naturally arises.

In recent years, function spaces, especially Hardy spaces and BMO spaces associated with different operators, have inspired great interest (see, e.g., [2], [4], [5], [15], [17], [16], [20], [22], [35], and references therein). In particular, Auscher, Duong, and McIntosh in [2] first introduced the Hardy space $H_{L}^{1}\left(\mathbb{R}^{n}\right)$ associated with an operator $L$ whose heat kernel satisfies a pointwise Poisson-type upper bound by means of a corresponding variant of the Lusin area function, and they also established its molecular characterization. Later, Duong and Yan in [17] and [16] introduced its dual space $\mathrm{BMO}_{L}\left(\mathbb{R}^{n}\right)$ and established the dual relation between $H_{L}^{1}\left(\mathbb{R}^{n}\right)$ and $\mathrm{BMO}_{L}\left(\mathbb{R}^{n}\right)$. Yan in [35] further generalized these results to the Hardy spaces $H_{L}^{p}\left(\mathbb{R}^{n}\right)$ with certain $p \leq 1$ and their dual spaces. Also, Auscher and Russ in [5] studied the Hardy space $H_{L}^{1}$ on strongly Lipschitz domains associated with a divergence form elliptic operator $L$ whose heat kernels have the Gaussian upper bounds and regularity. Very recently, Auscher, McIntosh, and Russ in [4] treated the Hardy space $H^{p}$ with $p \in[1, \infty]$ associated to the Hodge Laplacian on a Riemannian manifold with doubling measure. Meanwhile, Hofmann and Mayboroda in [22] further studied the Hardy space $H_{L}^{1}\left(\mathbb{R}^{n}\right)$ and its dual space adapted to a second-order divergence form elliptic operator $L$ on $\mathbb{R}^{n}$ with bounded complex coefficients, and these operators may not have the 
pointwise heat kernel bounds. Then Hofmann, Lu, D. Mitrea, M. Mitrea, and Yan in [20] introduced the new Hardy spaces $H_{L}^{p}, 1 \leq p<\infty$, on a metric space $X$ associated to a nonnegative self-adjoint operator $L$ satisfying Davies-Gaffney estimates. The motivation for investigating Hardy spaces, for example, is that boundedness in Hardy spaces can be interpolated with an $L^{2}$-boundedness to obtain other $L^{p}$-boundednesses. For this particular application, the atomic decomposition of Hardy spaces is very convenient and, as pointed out in some recent works, the set of atoms is even sufficient, so we do not have to study boundedness on the whole Hardy space (which may be difficult to prove; see, e.g., [8] and [10]).

On the other hand, as generalizations of Hardy spaces $H^{p}\left(\mathbb{R}^{n}\right)$, the OrliczHardy spaces on $\mathbb{R}^{n}$ and their dual spaces have received considerable attention as well. In particular, Strömberg [33] and Janson [23] introduced generalized Hardy spaces $H_{\omega}\left(\mathbb{R}^{n}\right)$, via replacing the norm $\|\cdot\|_{L^{p}\left(\mathbb{R}^{n}\right)}$ by the Orlicznorm $\|\cdot\|_{L(\omega)}$ in the definition of $H^{p}\left(\mathbb{R}^{n}\right)$, where $\omega$ is an Orlicz function on $[0, \infty)$ satisfying some control conditions. Viviani [34] further characterized these spaces $H_{\omega}$ on spaces of homogeneous type via atoms. The dual spaces of these spaces were also studied in [33], [23], [34], and [19]. Very recently, Orlicz-Hardy spaces associated with certain operators have been investigated by a number of mathematicians (see, e.g., [26], [24], [25], [29], and references therein). In particular, Jiang and Yang [24], [25] introduced the new Orlicz-Hardy spaces associated to divergence form elliptic operators and to nonnegative self-adjoint operators holding Davies-Gaffney estimates. Meanwhile, Liang, D. Yang, and S. Yang in [29] presented some applications of Orlicz-Hardy spaces associated with operators satisfying Poisson estimates.

Motivated by all of the above-mentioned facts, in the following we wish to allow generalized Orlicz-Hardy spaces related to generalized Orlicz functions. In this setting, the Orlicz function $\omega(t)$ is replaced by a function $\varphi(x, t)$, called the Musielak-Orlicz function (see [30], [14]), that may vary in the spatial variables and possesses some control conditions. We then introduce a new class of Hardy spaces $H_{\varphi, L}$, called Hardy spaces of MusielakOrlicz type associated to the operator $L$, and their dual spaces.

Before coming to the main content of this article, we first recall some notation and known facts on second-order divergence form elliptic operators on $\mathbb{R}^{n}$ defined by (1.2).

Following [22], set

$$
p_{L} \equiv \inf \left\{p \geq 1: \sup _{t>0}\left\|e^{-t L}\right\|_{L^{p}\left(\mathbb{R}^{n}\right) \rightarrow L^{p}\left(\mathbb{R}^{n}\right)}<\infty\right\}
$$


and

$$
\widetilde{p}_{L} \equiv \sup \left\{p \leq \infty: \sup _{t>0}\left\|e^{-t L}\right\|_{L^{p}\left(\mathbb{R}^{n}\right) \rightarrow L^{p}\left(\mathbb{R}^{n}\right)}<\infty\right\} .
$$

It was proved by Auscher in [1] that if $n=1,2$, then $p_{L}=1$ and $\widetilde{p}_{L}=\infty$, and that if $n \geq 3$, then $p_{L}<2 n /(n+2)$ and $\widetilde{p}_{L}>2 n /(n-2)$. One could detail other situations: for example, if the matrix $A$ is real-valued, then the heat kernel has Gaussian bounds and so $p_{L}=1$ and $\widetilde{p}_{L}=\infty$, due to [6, Theorem 4] or to the case of a higher-order operator (see [2, Section 7.2]).

For all $f \in L^{2}\left(\mathbb{R}^{n}\right)$ and $x \in \mathbb{R}^{n}$, define the Lusin area function by

$$
\mathcal{S}_{L} f(x) \equiv\left(\iint_{\Gamma(x)}\left|t^{2} L e^{-t^{2} L} f(y)\right|^{2} \frac{d y d t}{t^{n+1}}\right)^{1 / 2},
$$

where and in what follows, $\Gamma(x) \equiv\left\{(y, t) \in \mathbb{R}^{n} \times(0, \infty):|x-y|<t\right\}$.

This article is organized as follows.

In Section 2, we recall some notions and known results concerning operators associated with $L$, and we present some basic assumptions on the Musielak-Orlicz function $\omega$ considered here. Under these restrictions, the Musielak-Orlicz Hardy space $H_{\omega, L}\left(\mathbb{R}^{n}\right)$ behaves more closely like the classical Hardy space.

In Section 3, we introduce the tent spaces $T_{\omega}\left(\mathbb{R}_{+}^{n+1}\right)$ associated to $\omega$ and we establish its atomic characterization (see Theorem 3.1 below). By the proof of Theorem 3.1, we observe that if a function $f \in T_{\omega}\left(\mathbb{R}_{+}^{n+1}\right) \cap$ $T_{2}^{p}\left(\mathbb{R}_{+}^{n+1}\right), p \in(0, \infty)$, then there exists an atomic decomposition of $F$ which converges in both $T_{\omega}\left(\mathbb{R}_{+}^{n+1}\right)$ and $T_{2}^{p}\left(\mathbb{R}_{+}^{n+1}\right)$ (see Proposition 3.1 below). As a consequence, we show that if $f \in T_{\omega}\left(\mathbb{R}_{+}^{n+1}\right) \cap T_{2}^{2}\left(\mathbb{R}_{+}^{n+1}\right)$, then there exists an atomic decomposition of $f$ which converges in both $T_{\omega}\left(\mathbb{R}_{+}^{n+1}\right)$ and $T_{2}^{p}\left(\mathbb{R}_{+}^{n+1}\right)$ for all $p \in[1,2]$ (see Corollary 3.1 below). These convergences play a significant role throughout this paper.

In Section 4, we first introduce the Musielak-Orlicz Hardy space $H_{\omega, L}\left(\mathbb{R}^{n}\right)$, and then prove that the operator $\pi_{L, M}$, which is introduced in [16] and initially defined on $f \in L^{2}\left(\mathbb{R}_{+}^{n+1}\right)$ with compact support by

$$
\pi_{L, M} f \equiv C_{M} \int_{0}^{\infty}\left(t^{2} L\right)^{M+1} e^{-t^{2} L} f(\cdot, t) \frac{d t}{t},
$$

where $M \in \mathbb{N}$ and where

$$
C_{M} \int_{0}^{\infty} t^{2(M+2)} e^{-2 t^{2}} \frac{d t}{t}=1
$$


maps the tent space $T_{2}^{p}\left(\mathbb{R}_{+}^{n+1}\right)$ continuously into $L^{p}\left(\mathbb{R}^{n}\right)$ for $p \in\left(p_{L}, \widetilde{p}_{L}\right)$ and $T_{\omega}\left(\mathbb{R}_{+}^{n+1}\right)$ continuously into $H_{\omega, L}\left(\mathbb{R}^{n}\right)$ (see Proposition 4.1 below). As a result, we obtain a molecular decomposition for elements in $H_{\omega, L}\left(\mathbb{R}^{n}\right) \cap$ $L^{2}\left(\mathbb{R}^{n}\right)$ which converges in $L^{p}\left(\mathbb{R}^{n}\right)$ for $p \in\left(p_{L}, 2\right]$ (see Proposition 4.2 below). Due to this molecular decomposition of $H_{\omega, L}\left(\mathbb{R}^{n}\right)$, we further obtain the duality between $H_{\omega, L}\left(\mathbb{R}^{n}\right)$ and $\mathrm{BMO}_{\rho, L^{*}}\left(\mathbb{R}^{n}\right)$ (see Theorem 4.1 below). The rest of Section 4 is devoted to establishing the $\rho$-Carleson measure characterization (see Theorem 4.2 below) and the John-Nirenberg inequality (see Theorem 4.3 below) for the space $\mathrm{BMO}_{\rho, L}\left(\mathbb{R}^{n}\right)$.

In Section 5, as an application, we give some sufficient conditions which guarantee the boundedness of linear or nonnegative sublinear operators from $H_{\omega, L}\left(\mathbb{R}^{n}\right)$ to $L(\omega)$. In particular, we show that the Riesz transform $\nabla L^{-1 / 2}$ and the Littlewood-Paley $g$-function $g_{L}$ map $H_{\omega, L}\left(\mathbb{R}^{n}\right)$ continuously into $L(\omega)$ (see Theorem 5.1 below).

It should be pointed out that very recently the authors in [36] have investigated the Musielak-Orlicz Hardy spaces associated with operators. However, the approach in [36], which is mainly initiated by the work of Ky in [28], differs from our approach, which is strongly motivated by Hofmann and Mayboroda [22] and Jiang and Yang [24]. For instance, Musielak-Orlicz functions $\omega$ considered in [36] are growth functions that require that the functions $\omega(\cdot, t)$ belong to the uniform weight class $\mathbb{A}_{\infty}$ and satisfy the uniformly reverse Hölder condition, whereas we do not make such assumptions in our paper. Moreover, let $\mathcal{X}$ be a metric space with doubling measure; then, under the assumptions for the Musielak-Orlicz function similar to that in [36], the Musielak-Orlicz Hardy space, associated with a one-to-one operator of type $\omega$ having a bounded $H_{\infty}$ functional calculus in $L^{2}(\mathcal{X})$ and satisfying the reinforced off-diagonal estimates, was studied in [12]. As a special case of this setting, the Musielak-Orlicz Hardy space associated with divergence form elliptic operators with complex bounded measurable coefficients on $\mathbb{R}^{n}$ is also studied. However, the results in [12] and in this paper are different because the assumptions for the Musielak-Orlicz function are different, as indicated above.

It is believed that the results in this paper can be extended to the setting when the underlying spaces are of homogeneous type and operators satisfy bounded $H_{\infty}$ functional calculus and Davies-Gaffney estimates. This will be studied in a future article. 


\section{$\S 2$. Preliminaries}

In this section, we recall some notions and notation on the divergence form elliptic operator and on Musielak-Orlicz-type functions, and we introduce some basic assumptions on these functions. We first highlight some conventions. Throughout the current paper, $L$ always denotes the second-order divergence form operator as in (1.2). We denote by $C$ a positive constant which is independent of the main parameters, but which may vary from line to line. The symbol $X \lesssim Y$ means that there exists a positive constant $C$ such that $X \leq C Y$.

\subsection{Some notions on the divergence form elliptic operator $L$}

In this section, we review some of the standard facts on the operator $L$ considered in these pages.

A family $\left\{S_{t}\right\}_{t>0}$ of operators is said to satisfy the $L^{2}$ off-diagonal estimates, which are also called the Gaffney estimates (see [22]), if there exist positive constants $c, C$, and $\beta$ such that, for arbitrary closed sets $E, F \subset \mathbb{R}^{n}$,

$$
\left\|S_{t} f\right\|_{L^{2}(F)} \leq C e^{-\left(\frac{\operatorname{dist}(E, F)^{2}}{c t}\right)^{\beta}}\|f\|_{L^{2}(E)}
$$

for every $t>0$ and every $f \in L^{2}\left(\mathbb{R}^{n}\right)$ supported in $E$. In what follows, we will need the following important results which were obtained in [1], [3], [22], and [21].

LEMma 2.1 ([21, Lemma 2.3]). If two families of operators, $\left\{S_{t}\right\}_{t>0}$ and $\left\{T_{t}\right\}_{t>0}$, satisfy Gaffney estimates, then so does $\left\{S_{t} T_{t}\right\}_{t>0}$. Moreover, there exist positive constants $c, C$, and $\beta$ such that, for arbitrary closed sets $E, F \subset$ $\mathbb{R}^{n}$,

$$
\left\|S_{s} T_{t} f\right\|_{L^{2}(F)} \leq C e^{-\left(\frac{\operatorname{dist}(E, F)^{2}}{c \max \{s, t\}}\right)^{\beta}}\|f\|_{L^{2}(E)}
$$

for every $s, t>0$ and every $f \in L^{2}\left(\mathbb{R}^{n}\right)$ supported in $E$.

Lemma 2.2 ([3, Lemma 2.1], [21, Lemma 2.1]). The families

$$
\left\{e^{-t L}\right\}_{t>0}, \quad\left\{t L e^{-t L}\right\}_{t>0}, \quad\left\{t^{1 / 2} \nabla e^{-t L}\right\}_{t>0}
$$

as well as

$$
\left\{(I+t L)^{-1}\right\}_{t>0}, \quad\left\{t^{1 / 2} \nabla(I+t L)^{-1}\right\}_{t>0},
$$

are bounded on $L^{2}\left(\mathbb{R}^{n}\right)$ uniformly in $t$ and satisfy the Gaffney estimates with positive constants $c, C$ depending on $n, \lambda_{A}, \Lambda_{A}$ as in (1.1) only. For the operators in (2.1), $\beta=1$, while in (2.2), $\beta=1 / 2$. 
Lemma 2.3 ([1, Section 4], [22, Lemma 2.5]). There exist $c, C \in(0, \infty)$ such that

(i) for every $p$ and $q$ with $p_{L}<p \leq q<\widetilde{p}_{L}$, the families $\left\{e^{-t L}\right\}_{t>0}$ and $\left\{t L e^{-t L}\right\}_{t>0}$ satisfy $L^{p}-L^{q}$ off-diagonal estimates; that is, for arbitrary closed sets $E, F \subset \mathbb{R}^{n}$,

$$
\left\|e^{-t L} f\right\|_{L^{q}(F)}+\left\|t L e^{-t L} f\right\|_{L^{q}(F)} \leq C t^{\frac{n}{2}\left(\frac{1}{q}-\frac{1}{p}\right)} e^{-\frac{\operatorname{dist}(E, F)^{2}}{c t}}\|f\|_{L^{p}(E)}
$$

for every $t>0$ and every $f \in L^{p}\left(\mathbb{R}^{n}\right)$ supported in $E$, and thus the operators $\left\{e^{-t L}\right\}_{t>0}$ and $\left\{t L e^{-t L}\right\}_{t>0}$ are bounded from $L^{p}\left(\mathbb{R}^{n}\right)$ to $L^{q}\left(\mathbb{R}^{n}\right)$ with the norm $C t^{\frac{n}{2}\left(\frac{1}{q}-\frac{1}{p}\right)}$;

(ii) for every $p \in\left(p_{L}, \widetilde{p}_{L}\right)$, the family $\left\{(I+t L)^{-1}\right\}_{t>0}$ satisfies $L^{p}-L^{p}$ offdiagonal estimates; that is, for arbitrary closed sets $E, F \subset \mathbb{R}^{n}$,

$$
\left\|(I+t L)^{-1} f\right\|_{L^{q}(F)} \leq C t^{\frac{n}{2}\left(\frac{1}{q}-\frac{1}{p}\right)} e^{-\frac{\operatorname{dist}(E, F)}{c t^{1 / 2}}}\|f\|_{L^{p}(E)}
$$

for every $t>0$ and every $f \in L^{p}\left(\mathbb{R}^{n}\right)$ supported in $E$.

Lemma 2.4 ([22, Lemma 2.6]). Let $k \in \mathbb{N}$ and $p \in\left(p_{L}, \widetilde{p}_{L}\right)$. Then the operator given for any $f \in L^{p}\left(\mathbb{R}^{n}\right)$ and any $x \in \mathbb{R}^{n}$,

$$
\mathcal{S}_{L}^{k} f(x) \equiv\left(\iint_{\Gamma(x)}\left|\left(t^{2} L\right)^{k} e^{-t^{2} L} f(y)\right|^{2} \frac{d y d t}{t^{n+1}}\right)^{1 / 2},
$$

is bounded on $L^{p}\left(\mathbb{R}^{n}\right)$.

\subsection{Musielak-Orlicz-type functions}

Let us first present some notions on Musielak-Orlicz-type functions.

A function $\omega:[0, \infty) \rightarrow[0, \infty)$ is called an Orlicz function if it is nondecreasing and if $\omega(0)=0 ; \omega(t)>0, t>0 ; \lim _{t \rightarrow \infty} \omega(t)=\infty$. A function $\omega: \mathbb{R}^{n} \times[0, \infty) \rightarrow[0, \infty)$ is called a Musielak-Orlicz function if the function $\omega(x, \cdot):[0, \infty) \rightarrow[0, \infty)$ is an Orlicz function for all $x \in \mathbb{R}^{n}$ and the function $\omega(\cdot, t)$ is a measurable function for all $t \in[0, \infty)$.

Let $\omega$ be a Musielak-Orlicz function. The function $\omega$ is said to be of uniformly upper-type $p$ (resp., uniformly lower-type $p$ ) for certain $p \in[0, \infty)$ if there exists a positive constant $C$ such that for all $x \in \mathbb{R}^{n}$, all $t \geq 1$ (resp., $t \in(0,1])$ and all $s \in(0, \infty)$,

$$
\omega(x, s t) \leq C t^{p} \omega(x, s) .
$$

If $\omega$ is of both uniformly upper-type $p_{1}$ and lower-type $p_{0}$, then $\omega$ is said to be of type $\left(p_{0}, p_{1}\right)$. A typical example of such $\omega$ is

$$
\omega(x, t):=f(x) g(t)
$$


for all $x \in \mathbb{R}^{n}$ and all $t \in[0, \infty)$, where $f$ is a positive measurable function on $\mathbb{R}^{n}$ and where $g$ is an Orlicz function on $[0, \infty)$ of upper-type $p_{1}$ and lower-type $p_{0}$. Another example of Musielak-Orlicz function $\omega$ of uniformly upper-type $p \in(0,1]$ is, for instance,

$$
\omega(x, t)=\frac{t^{p}}{f(x)+[\log (e+t)]^{\alpha}},
$$

where $\alpha \in[0,2 p(1+\log 2)]$ and $f$ is a positive measurable function on $\mathbb{R}^{n}$. It is also interesting to observe that if

$$
\omega(x, t)=\frac{t^{p}}{f(x)+g(t)},
$$

where $f$ is a positive measurable function on $\mathbb{R}^{n}$ and where $g$ is a decreasing positive function on $[0, \infty)$, then $\omega$ is a Musielak-Orlicz function of uniformly lower-type $p$.

Let

$$
\begin{gathered}
p_{\omega}^{+} \equiv \inf \{p>0 \text { : there exists } C>0 \text { such that }(2.3) \\
\text { holds for all } \left.x \in \mathbb{R}^{n}, t \in[1, \infty), s \in(0, \infty)\right\},
\end{gathered}
$$

and let

$$
\begin{aligned}
p_{\omega}^{-} \equiv & \sup \{p>0: \text { there exists } C>0 \text { such that }(2.3) \\
& \text { holds for all } \left.x \in \mathbb{R}^{n}, t \in(0,1], s \in(0, \infty)\right\} .
\end{aligned}
$$

The function $\omega$ is said to be of strictly uniformly lower-type $p$ if, for all $x \in \mathbb{R}^{n}, t \in(0,1)$, and $s \in(0, \infty), \omega(x, s t) \leq t^{p} \omega(x, s)$ and if

$$
\begin{aligned}
& p_{\omega} \equiv \sup \left\{p>0: \omega(x, s t) \leq t^{p} \omega(x, s) \text { holds for all } x \in \mathbb{R}^{n},\right. \\
& \quad s \in(0, \infty) \text { and } t \in(0,1)\} .
\end{aligned}
$$

It is easy to see that $p_{\omega} \leq p_{\omega}^{-} \leq p_{\omega}^{+}$for all $\omega$. In what follows, $p_{\omega}, p_{\omega}^{-}$, and $p_{\omega}^{+}$ are called the strictly critical lower-type index, the critical lower-type index, and the critical upper-type index of $\omega$, respectively.

REMARK 2.1. We see that if $p_{\omega}$ is defined as above, then $\omega$ is also of strictly uniformly lower-type $p_{\omega}$. In other words, $p_{\omega}$ is attainable.

Throughout this article, we always assume that $\omega$ satisfies the following assumptions. 
Assumption (A). Suppose that $\omega$ is a Musielak-Orlicz function which is of uniformly upper-type 1 and for which $p_{\omega} \in(0,1]$. In addition, for every $x \in \mathbb{R}^{n}, \omega(x, \cdot)$ is continuous, strictly increasing on $\mathbb{R}_{+}$.

Note that if $\omega$ satisfies Assumption (A), then it has the following properties (see [28, Lemma 4.1] for its proof).

\section{LEMMA 2.5.}

(i) The function $\omega$ is uniformly $\sigma$-quasi-subadditive on $\mathbb{R}^{n} \times[0, \infty)$; namely, there exists a positive constant $C$ such that for all $\left(x, t_{j}\right) \in \mathbb{R}^{n} \times[0, \infty)$ with $j \in \mathbb{Z}_{+}, \omega\left(x, \sum_{j=1}^{\infty} t_{j}\right) \leq C \sum_{j=1}^{\infty} \omega\left(x, t_{j}\right)$.

(ii) Let $\widetilde{\omega}(x, t):=\int_{0}^{t} \frac{\omega(x, s)}{s} d s$ for all $(x, t) \in \mathbb{R}^{n} \times[0, \infty)$. Then $\widetilde{\omega}$ is equivalent to $\omega$; moreover, $\widetilde{\omega}$ also satisfies Assumption $(A)$.

Convention (B). From Assumption (A), it follows that $0<p_{\omega} \leq p_{\omega}^{-} \leq$ $p_{\omega}^{+} \leq 1$. In what follows, if $(2.3)$ holds for $p_{\omega}^{+}$with $t \in[1, \infty)$, then we choose $\widetilde{p}_{\omega} \equiv p_{\omega}^{+}$; otherwise $p_{\omega}^{+}<1$ and we choose $\widetilde{p}_{\omega} \in\left(p_{\omega}^{+}, 1\right)$ to be close enough to $p_{\omega}^{+}$.

Let $\omega$ satisfy Assumption (A). A measurable function $f$ on $\mathbb{R}^{n}$ is said to be in the Lebesgue-type space $L(\omega)$ if

$$
\int_{\mathbb{R}^{n}} \omega(x,|f(x)|) d x<\infty .
$$

Moreover, for any $f \in L(\omega)$, define

$$
\|f\|_{L(\omega)} \equiv \inf \left\{\lambda>0: \int_{\mathbb{R}^{n}} \omega\left(x, \frac{|f(x)|}{\lambda}\right) d x \leq 1\right\} .
$$

The function $\rho$ defined below plays an important role in this paper.

Definition 2.1. For each $x \in \mathbb{R}^{n}$, we define the function $\omega^{-1}(x, \cdot)$ and $\rho(x, \cdot)$ on $\mathbb{R}_{+}$as follows:

$$
\omega^{-1}(x, t) \equiv \sup \{s \geq 0: \omega(x, s) \leq t\}
$$

and

$$
\rho(x, t) \equiv \frac{t^{-1}}{\omega^{-1}\left(x, t^{-1}\right)} .
$$


Then it is easy to see that $\omega^{-1}(x, \cdot)$ is continuous, strictly increasing, and that for every $x \in \mathbb{R}^{n}$,

$$
\omega^{-1}(x, \omega(x, t))=t
$$

and

$$
\omega\left(x, \omega^{-1}(x, t)\right)=t .
$$

Moreover, the types of $\omega$ and $\omega^{-1}$ have the following relation.

Lemma 2.6. Let $0<p \leq q \leq 1$. If $\omega$ is of type $(p, q)$, then $\omega^{-1}$ is of type $\left(q^{-1}, p^{-1}\right)$.

Proof. By symmetry, it suffices to show that if $\omega$ is of uniformly lowertype $p$, then $\omega^{-1}$ is of uniformly upper-type $p^{-1}$. Suppose that there exists a constant $C \geq 1$ such that for all $x \in \mathbb{R}^{n}, s \geq 0, t \leq 1$,

$$
\omega(x, s t) \leq C t^{p} \omega(x, s) .
$$

Then for any $x \in \mathbb{R}^{n}, s \geq 0, t \geq 1$, and $u \geq 0$ such that $\omega(x, u) \leq s t$, it follows from (2.6) that

$$
\omega\left(x, \frac{u}{t^{\frac{1}{p}}}\right) \leq \frac{C \omega(x, u)}{t} \leq C s .
$$

This implies that

$$
u \leq t^{\frac{1}{p}} \omega^{-1}(x, C s)
$$

and hence

$$
\omega^{-1}(x, s t) \leq t^{\frac{1}{p}} \omega^{-1}(x, C s) .
$$

On the other hand, observe that

$$
\omega^{-1}(x, C s)=\sup \left\{\lambda \geq 0: \frac{\omega(x, \lambda)}{C} \leq s\right\}
$$

and, by (2.6), for any $\lambda \geq 0$,

$$
\omega\left(x, \frac{\lambda}{C^{\frac{2}{p}}}\right) \leq \frac{\omega(x, \lambda)}{C} ;
$$

then we deduce that

$$
\omega^{-1}(x, C s) \leq C^{\frac{2}{p}} \omega^{-1}(x, s),
$$

which together with (2.7) completes the proof of Lemma 2.6.

Assumption (C). Let $\omega$ satisfy Assumption (A). Moreover, suppose that

(i) there exist positive constants $C_{1}, C_{2}$ such that for any $x \in \mathbb{R}^{n}, C_{1} \leq$ $\omega(x, 1) \leq C_{2}$ 
(ii) there exists a positive constant $C$ such that for any locally integrable positive function $f$ on $\mathbb{R}^{n}$, for any ball $B$ in $\mathbb{R}^{n}$,

$$
\frac{1}{|B|} \int_{B} \omega(x, f(x)) d x \leq C \inf _{x \in \mathbb{R}^{n}} \omega\left(x, \frac{1}{|B|} \int_{B} f(x) d x\right) .
$$

A typical example of a Musielak-Orlicz function $\omega$ that satisfies Assumption $(\mathrm{C})$ is $\omega(x, t)=h(x) \varphi(t)$ for all $x \in \mathbb{R}^{n}$ and $t \in[0, \infty)$, where $h$ is a positive measurable function on $\mathbb{R}^{n}$ so that there exist positive constants $C_{1}, C_{2}$ such that, for any $x \in \mathbb{R}^{n}, C_{1} \leq h(x) \leq C_{2}$ and $\varphi$ is a continuous, increasing, and concave Orlicz function on $[0, \infty)$ with $p_{\varphi} \in(0,1]$. Besides, there exist Musielak-Orlicz functions which are not of that form and that satisfy Assumption (C), for instance, $\omega(x, t)=\frac{t}{h(x)+[\log (e+t)]^{\alpha}}$, where $\alpha \in$ $[0,2(1+\log 2)]$ and where $h$ is a positive measurable function on $\mathbb{R}^{n}$ such that there exist positive constants $C_{1}, C_{2}$ for any $x \in \mathbb{R}^{n}, C_{1} \leq h(x) \leq C_{2}$.

\section{$\S 3$. Tent spaces associated to Musielak-Orlicz functions}

In this section, we will deal with the tent spaces associated to MusielakOrlicz functions. Let us first recall some notions.

For any $\nu>0$ and $x \in \mathbb{R}^{n}$, let $\mathbb{R}_{+}^{n+1} \equiv \mathbb{R}^{n} \times(0, \infty)$, and let

$$
\Gamma_{\nu}(x) \equiv\left\{(y, t) \in \mathbb{R}_{+}^{n+1}:|x-y|<\nu t\right\}
$$

denote the cone of aperture $\nu$ with vertex $x \in \mathbb{R}^{n}$. For any closed set $F$ of $\mathbb{R}^{n}$, denote by $\mathcal{R}_{\nu} F$ the union of all cones with vertices in $F$, that is, $\mathcal{R}_{\nu} F \equiv \bigcup_{x \in F} \Gamma_{\nu}(x)$; and for any open set $O$ in $\mathbb{R}^{n}$, denote the tent over $O$ by $T_{\nu}(O)$, which is defined as $T_{\nu}(O) \equiv\left[\mathcal{R}_{\nu}\left(O^{\complement}\right)\right]^{\complement}$. Note that

$$
T_{\nu}(O)=\left\{(x, t) \in \mathbb{R}^{n} \times(0, \infty): \operatorname{dist}\left(x, O^{\complement}\right) \geq \nu t\right\} .
$$

In what follows, we denote $\Gamma_{1}(x), \mathcal{R}_{1}(F)$, and $T_{1}(O)$ simply by $\Gamma(x), \mathcal{R}(F)$, and $\widehat{O}$, respectively.

Let $F$ be a closed subset of $\mathbb{R}^{n}$, and let $O \equiv F^{\complement}$. Assume that $|O|<\infty$. For any fixed $\gamma \in(0,1)$, we say that $x \in \mathbb{R}^{n}$ has the global $\gamma$-density with respect to $F$ if

$$
\frac{|B(x, r) \cap F|}{|B(x, r)|} \geq \gamma
$$

for all $r>0$. Denote by $F^{*}$ the set of all such $x$. Obviously, $F^{*}$ is a closed subset of $F$. Let $O^{*} \equiv\left(F^{*}\right)^{\complement}$. Then it is easy to see that $O \subset O^{*}$. In fact, we have

$$
O^{*}=\left\{x \in \mathbb{R}^{n}: \mathcal{M}\left(\chi_{O}\right)(x)>1-\gamma\right\}
$$


where $\mathcal{M}$ denotes the Hardy-Littlewood maximal function on $\mathbb{R}^{n}$. As a result, by the weak type $(1,1)$ of $\mathcal{M}$, we have $\left|O^{*}\right| \leq C(\gamma)|O|$, where $C(\gamma)$ denotes a positive constant depending on $\gamma$.

The proof of the following lemma is similar to that of [13, Lemma 2]; we omit the details.

Lemma 3.1. Let $\nu, \eta \in(0, \infty)$. Then there exist positive constants $\gamma \in$ $(0,1)$ and $C(\gamma, \nu, \eta)$ such that, for any closed subset $F$ of $\mathbb{R}^{n}$ whose complement has finite measure and any nonnegative measurable function $H$ on $\mathbb{R}_{+}^{n+1}$,

$$
\iint_{\mathcal{R}_{\nu}\left(F^{*}\right)} H(y, t) t^{n} d y d t \leq C(\gamma, \nu, \eta) \int_{F}\left\{\iint_{\Gamma_{\eta}(x)} H(y, t) d y d t\right\} d x
$$

where $F^{*}$ denotes the set of points in $\mathbb{R}^{n}$ with global $\gamma$-density with respect to $F$.

Let $\nu \in(0, \infty)$. For all measurable functions $g$ on $\mathbb{R}_{+}^{n+1}$ and all $x \in \mathbb{R}^{n}$, let

$$
\mathcal{A}_{\nu}(g)(x) \equiv\left(\iint_{\Gamma_{\nu}(x)}|g(y, t)|^{2} \frac{d y d t}{t^{n+1}}\right)^{1 / 2}
$$

and denote $\mathcal{A}_{1}(g)$ simply by $\mathcal{A}(g)$.

Coifman, Meyer, and Stein in [13] introduced the tent space $T_{2}^{p}\left(\mathbb{R}_{+}^{n+1}\right)$ for $p \in(0, \infty)$, which is defined as the space of all measurable functions $g$ such that $\|g\|_{T_{2}^{p}\left(\mathbb{R}_{+}^{n+1}\right)} \equiv\|\mathcal{A}(g)\|_{L^{p}\left(\mathbb{R}^{n}\right)}<\infty$.

Now let $\omega$ satisfy Assumption $(\mathrm{A})$. Then we define the tent space $T_{\omega}\left(\mathbb{R}_{+}^{n+1}\right)$ associated to the function $\omega$ as the space of measurable functions $g$ on $\mathbb{R}_{+}^{n+1}$ such that $\mathcal{A}(g) \in L(\omega)$ with the norm defined by

$$
\|g\|_{T_{\omega}\left(\mathbb{R}_{+}^{n+1}\right)} \equiv\|\mathcal{A}(g)\|_{L(\omega)}=\inf \left\{\lambda>0: \int_{\mathbb{R}^{n}} \omega\left(x, \frac{\mathcal{A}(g)(x)}{\lambda}\right) d x \leq 1\right\}
$$

Let $p \in(1, \infty)$, let $\omega$ satisfy Assumption (C), and let $\rho$ be the function defined by $(2.5)$. A function $a$ on $\mathbb{R}_{+}^{n+1}$ is called an $(\omega, p)$-atom if

(i) there exists a ball $B \subset \mathbb{R}^{n}$ such that supp $a \subset \widehat{B}$;

(ii) $\|a\|_{T_{2}^{p}\left(\mathbb{R}_{+}^{n+1}\right)} \leq|B|^{\frac{1}{p}-1} \inf _{x \in B}[\rho(x,|B|)]^{-1}$.

Furthermore, if $a$ is an $(\omega, p)$-atom for all $p \in(1, \infty)$, we then call $a$ an $(\omega, \infty)$-atom. 


\section{REMARK 3.1.}

(i) It is not difficult to verify that for a function $\omega$ satisfying Assumption (C), there exist positive constants $K_{1}, K_{2}$ such that for any $x \in \mathbb{R}^{n}$, $K_{1} \leq \omega^{-1}(x, 1) \leq K_{2}$, and hence $\inf _{x \in B}[\rho(x,|B|)]^{-1}$ is strictly positive.

(ii) In addition, for all $(\omega, p)$-atoms $a$, we have $\|a\|_{T_{\omega}\left(\mathbb{R}_{+}^{n+1}\right)} \lesssim 1$.

We are now ready to obtain the atomic characterization of the tent space $T_{\omega}\left(\mathbb{R}_{+}^{n+1}\right)$.

Theorem 3.1. Let $\omega$ satisfy Assumption $(C)$. Then for any $f \in T_{\omega}\left(\mathbb{R}_{+}^{n+1}\right)$, there exist $(\omega, \infty)$-atoms $\left\{a_{j}\right\}_{j=1}^{\infty}$ and numbers $\left\{\lambda_{j}\right\}_{j=1}^{\infty} \subset \mathbb{C}$ such that for almost every $(x, t) \in \mathbb{R}_{+}^{n+1}$,

$$
f(x, t)=\sum_{j=1}^{\infty} \lambda_{j} a_{j}(x, t) .
$$

Moreover, there exists a positive constant $C$ such that for all $f \in T_{\omega}\left(\mathbb{R}_{+}^{n+1}\right)$,

$$
\begin{aligned}
\Lambda\left(\left\{\lambda_{j} a_{j}\right\}_{j}\right) & \equiv \inf \left\{\lambda>0: \sum_{j=1}^{\infty}\left|B_{j}\right| \inf _{x \in \mathbb{R}^{n}} \omega\left(x, \frac{\left|\lambda_{j}\right|}{\lambda\left|B_{j}\right| \sup _{x \in B_{j}} \rho\left(x,\left|B_{j}\right|\right)}\right) \leq 1\right\} \\
& \leq C\|f\|_{T_{\omega}\left(\mathbb{R}_{+}^{n+1}\right)}
\end{aligned}
$$

where $\widehat{B_{j}}$ appears as the support of $a_{j}$.

Proof. We prove this theorem by exploiting some ideas found in [13, proof of Theorem 1] and [24, Theorem 3.1]. Let $f \in T_{\omega}\left(\mathbb{R}_{+}^{n+1}\right)$. For any $k \in \mathbb{Z}$, let $O_{k} \equiv\left\{x \in \mathbb{R}^{n}: \mathcal{A}(f)(x)>2^{k}\right\}$ and $F_{k} \equiv\left(O_{k}\right)^{\complement}$. Since $f \in T_{\omega}\left(\mathbb{R}_{+}^{n+1}\right)$, for each $k, O_{k}$ is an open set and $\left|O_{k}\right|<\infty$.

Since $\omega$ is of uniformly upper-type 1 and $1 \lesssim \omega(x, 1)$, by Lemma 3.1, for $k \in \mathbb{Z}$ and $k \leq 0$, we have

$$
\begin{aligned}
\iint_{\mathcal{R}\left(F_{k}^{*}\right)}|f(y, t)|^{2} \frac{d y d t}{t} & \lesssim \int_{F_{k}} \iint_{\Gamma(x)}|f(y, t)|^{2} \frac{d y d t}{t^{n+1}} d x \\
& \lesssim \int_{F_{k}} \mathcal{A}(f)(x)^{2} d x \lesssim \int_{F_{k}} \omega(x, \mathcal{A}(f)(x)) d x \rightarrow 0
\end{aligned}
$$

as $k \rightarrow-\infty$, which implies that $f=0$ almost everywhere in $\bigcap_{k \in \mathbb{Z}} \mathcal{R}\left(F_{k}^{*}\right)$, and hence, supp $f \subset\left\{\bigcup_{k \in \mathbb{Z}} \widehat{O_{k}^{*}} \cup E\right\}$, where $E \subset \mathbb{R}_{+}^{n+1}$ and $\iint_{E} \frac{d x d t}{t}=0$. 
Thus, for each $k$, by applying the Whitney decomposition to the set $O_{k}^{*}$, we obtain a set $I_{k}$ of indices and a family $\left\{Q_{k, j}\right\}_{j \in I_{k}}$ of disjoint cubes such that

(i) $\bigcup_{j \in I_{k}} Q_{k, j}=O_{k}^{*}$, and if $i \neq j$, then $Q_{k, j} \cap Q_{k, i}=\emptyset$; and

(ii) $\sqrt{n} \ell\left(Q_{k, j}\right) \leq \operatorname{dist}\left(Q_{k, j},\left(O_{k}^{*}\right)^{\complement}\right) \leq 4 \sqrt{n} \ell\left(Q_{k, j}\right)$, where $\ell\left(Q_{k, j}\right)$ denotes the side length of $Q_{k, j}$.

Next, for each $j \in I_{k}$, we choose a ball $B_{k, j}$ with the same center as $Q_{k, j}$ and with radius $\frac{11}{2} \sqrt{n}$-times $\ell\left(Q_{k, j}\right)$. Let $A_{k, j} \equiv \widehat{B_{k, j}} \cap\left(Q_{k, j} \times(0, \infty)\right) \cap\left(\widehat{O_{k}^{*}} \backslash\right.$ $\left.\widehat{O_{k+1}^{*}}\right)$, let

$$
a_{k, j} \equiv 2^{-k}\left|B_{k, j}\right|^{-1} \inf _{x \in B_{k, j}}\left[\rho\left(x,\left|B_{k, j}\right|\right)\right]^{-1} f \chi_{A_{k, j}}
$$

and let $\lambda_{k, j} \equiv 2^{k}\left|B_{k, j}\right| \sup _{x \in B_{k, j}}\left[\rho\left(x,\left|B_{k, j}\right|\right)\right]$. Note that $\left\{\left(Q_{k, j} \times(0, \infty)\right) \cap\right.$ $\left.\left(\widehat{O_{k}^{*}} \backslash \widehat{O_{k+1}^{*}}\right)\right\} \subset \widehat{B_{k, j}}$. By this, we conclude that $f=\sum_{k \in \mathbb{Z}} \sum_{j \in I_{k}} \lambda_{k, j} a_{k, j}$ almost everywhere.

Let us show that for each $k \in \mathbb{Z}$ and each $j \in I_{k}, a_{k, j}$ is an $(\omega, \infty)$ atom supported in $\widehat{B}_{k, j}$. Let $p \in(1, \infty), q \equiv p^{\prime}$ be the conjugate index of $p$, that is, $\frac{1}{q}+\frac{1}{p}=1$, and let $h \in T_{2}^{q}\left(\mathbb{R}_{+}^{n+1}\right)$ with $\|h\|_{T_{2}^{q}\left(\mathbb{R}_{+}^{n+1}\right)} \leq 1$. Since $A_{k, j} \subset\left(\widehat{O_{k+1}^{*}}\right)^{\complement}=\mathcal{R}\left(F_{k+1}^{*}\right)$, by Lemma 3.1 and the Hölder inequality, we have

$$
\begin{aligned}
\left|\left\langle a_{k, j}, h\right\rangle\right| \leq & \iint_{\mathbb{R}_{+}^{n+1}}\left|\left(a_{k, j} \chi_{A_{k, j}}\right)(y, t) h(y, t)\right| \frac{d y d t}{t} \\
\lesssim & \int_{F_{k+1}} \iint_{\Gamma(x)}\left|a_{k, j}(y, t) h(y, t)\right| \frac{d y d t}{t^{n+1}} d x \\
\lesssim & \int_{\left(O_{k+1}\right)^{\complement}} \mathcal{A}\left(a_{k, j}\right)(x) \mathcal{A}(h)(x) d x \\
\lesssim & 2^{-k}\left|B_{k, j}\right|^{-1} \inf _{x \in B_{k, j}}\left[\rho\left(x,\left|B_{k, j}\right|\right)\right]^{-1} \\
& \times\left(\int_{B_{k, j} \cap O_{k+1}^{\complement}}[\mathcal{A}(f)(x)]^{p} d x\right)^{\frac{1}{p}}\|h\|_{T_{2}^{q}\left(\mathbb{R}_{+}^{n+1}\right)} \\
\lesssim & \left|B_{k, j}\right|^{\frac{1}{p}-1} \inf _{x \in B_{k, j}}\left[\rho\left(x,\left|B_{k, j}\right|\right)\right]^{-1}
\end{aligned}
$$

which implies that $a_{k, j}$ is an $(\omega, p)$-atom supported in $\widehat{B}_{k, j}$ for all $p \in(1, \infty)$, and hence, an $(\omega, \infty)$-atom. 
On the other hand, for any $\lambda>0$, by Lemma 2.5 we further obtain

$$
\begin{aligned}
\sum_{k \in \mathbb{Z}} & \sum_{j \in I_{k}}\left|B_{k, j}\right| \inf _{x \in \mathbb{R}^{n}} \omega\left(x, \frac{\left|\lambda_{k, j}\right|}{\lambda\left|B_{k, j}\right| \sup _{x \in B_{k, j}} \rho\left(x,\left|B_{k, j}\right|\right)}\right) \\
& \lesssim \sum_{k \in \mathbb{Z}} \sum_{j \in I_{k}}\left|Q_{k, j}\right| \inf _{x \in \mathbb{R}^{n}} \omega\left(x, \frac{2^{k}}{\lambda}\right) \\
& \lesssim \sum_{k \in \mathbb{Z}}\left|O_{k}^{*}\right| \inf _{x \in \mathbb{R}^{n}} \omega\left(x, \frac{2^{k}}{\lambda}\right) \lesssim \sum_{k \in \mathbb{Z}}\left|O_{k}\right| \inf _{x \in \mathbb{R}^{n}} \omega\left(x, \frac{2^{k}}{\lambda}\right) \\
& \lesssim \sum_{k \in \mathbb{Z}} \int_{O_{k}} \inf _{x \in \mathbb{R}^{n}} \omega\left(x, \frac{2^{k}}{\lambda}\right) d x \lesssim \int_{\mathbb{R}^{n}} \sum_{k<\log _{2}[\mathcal{A}(f)(x)]} \inf _{x \in \mathbb{R}^{n}} \omega\left(x, \frac{2^{k}}{\lambda}\right) d x \\
& \lesssim \int_{\mathbb{R}^{n}} \sum_{k<\log _{2}[\mathcal{A}(f)(x)]} \int_{2^{k}}^{2^{k+1}} \inf _{x \in \mathbb{R}^{n}} \omega\left(x, \frac{t}{\lambda}\right) \frac{d t}{t} d x \\
& \lesssim \int_{\mathbb{R}^{n}} \int_{0}^{\frac{2 \mathcal{A}(f)(x)}{\lambda}} \omega(x, t) \frac{d t}{t} d x \lesssim \int_{\mathbb{R}^{n}} \omega\left(x, \frac{\mathcal{A}(f)(x)}{\lambda}\right) d x,
\end{aligned}
$$

which implies that (3.2) holds, and hence, completes the proof of Theorem 3.1 .

REMARK 3.2. Let $\left\{\lambda_{j}^{i}\right\}_{i, j} \subset \mathbb{C}$ and $\left\{a_{j}^{i}\right\}_{i, j}$ be $(\omega, p)$-atoms for certain $p \in$ $(1, \infty)$, where $i=1,2$. If $\sum_{j} \lambda_{j}^{1} a_{j}^{1}, \sum_{j} \lambda_{j}^{2} a_{j}^{2} \in T_{\omega}\left(\mathbb{R}_{+}^{n+1}\right)$, then by the fact that $\omega$ is subadditive and of strictly uniformly lower-type $p_{\omega}$, we have

$$
\left[\Lambda\left(\left\{\lambda_{j}^{i} a_{j}^{i}\right\}_{i, j}\right)\right]^{p_{\omega}} \leq \sum_{i=1}^{2}\left[\Lambda\left(\left\{\lambda_{j}^{i} a_{j}^{i}\right\}_{j}\right)\right]^{p_{\omega}} .
$$

Let $p \in(0,1]$ and $q \in(p, \infty) \cap[1, \infty)$. Recall that a function $a$ on $\mathbb{R}_{+}^{n+1}$ is called a $(p, q)$-atom if

(i) there exists a ball $B \subset \mathbb{R}^{n}$ such that supp $a \subset \widehat{B}$;

(ii) $\|a\|_{T_{2}^{q}\left(\mathbb{R}_{+}^{n+1}\right)} \leq|B|^{\frac{1}{q}-\frac{1}{p}}$.

We have the following convergence result.

Proposition 3.1. Let $\omega$ satisfy Assumption $(C)$, and let $p \in(0, \infty)$. If $f \in\left(T_{\omega}\left(\mathbb{R}_{+}^{n+1}\right) \cap T_{2}^{p}\left(\mathbb{R}_{+}^{n+1}\right)\right)$, then the decomposition (3.1) holds in both $T_{\omega}\left(\mathbb{R}_{+}^{n+1}\right)$ and $T_{2}^{p}\left(\mathbb{R}_{+}^{n+1}\right)$.

Proof. The proof that (3.1) holds in $T_{2}^{p}\left(\mathbb{R}_{+}^{n+1}\right)$ is analogous to that of $[24$, Theorem 3.1] and we omit the details. We only need to show that (3.1) holds 
in $T_{\omega}\left(\mathbb{R}_{+}^{n+1}\right)$. In fact, by the Hölder inequality, for each $k \in \mathbb{Z}$ and $j \in I_{k}$, we have

$$
\begin{aligned}
\frac{1}{\left|B_{k, j}\right|} \int_{\mathbb{R}^{n}} \mathcal{A}\left(\lambda_{k, j} a_{k, j}\right)(x) d x & \leq \frac{\left|\lambda_{k, j}\right|}{\left|B_{k, j}\right| 1 / 2}\left\|a_{k, j}\right\|_{T_{2}^{2}\left(\mathbb{R}_{+}^{n+1}\right)} \\
& \leq \frac{\left|\lambda_{k, j}\right|}{\left|B_{k, j}\right| \sup _{x \in B_{k, j}} \rho\left(x,\left|B_{k, j}\right|\right)} .
\end{aligned}
$$

From this together with the subadditive property of $\omega$ and $\mathcal{A}$, it follows that

$$
\begin{aligned}
& \int_{\mathbb{R}^{n}} \omega\left(x, \mathcal{A}\left(f-\sum_{|k|+|j| \leq N} \lambda_{k, j} a_{k, j}\right)(x)\right) d x \\
& \leq \sum_{|k|+|j|>N} \int_{\mathbb{R}^{n}} \omega\left(x, \mathcal{A}\left(\lambda_{k, j} a_{k, j}\right)(x)\right) d x \\
& \quad \lesssim \sum_{|k|+|j|>N}\left|B_{k, j}\right| \inf _{x \in \mathbb{R}^{n}} \omega\left(x, \frac{\left|\lambda_{k, j}\right|}{\left|B_{k, j}\right| \sup _{x \in B_{k, j}} \rho\left(x,\left|B_{k, j}\right|\right)}\right) \rightarrow 0,
\end{aligned}
$$

as $N \rightarrow \infty$, by (3.3). Now for any $\epsilon>0$, by the fact that $\omega$ is of uniformly upper-type 1 and (3.4), there exists $N_{0} \in \mathbb{N}$ such that when $N>N_{0}$,

$$
\int_{\mathbb{R}^{n}} \omega\left(x, \frac{1}{\epsilon} \mathcal{A}\left[f-\sum_{|k|+|j| \leq N} \lambda_{k, j} a_{k, j}\right](x)\right) d x \leq 1,
$$

which implies that when $N>N_{0},\left\|f-\sum_{|k|+|j| \leq N} \lambda_{k, j} a_{k, j}\right\|_{T_{\omega}\left(\mathbb{R}_{+}^{n+1}\right)} \leq \epsilon$. Thus, (3.1) holds in $T_{\omega}\left(\mathbb{R}_{+}^{n+1}\right)$, which completes the proof of Proposition 3.1.

As a consequence of Proposition 3.1, we have the following corollary which plays a significant role in this paper.

Corollary 3.1. Let $\omega$ satisfy Assumption (C). If $f \in T_{\omega}\left(\mathbb{R}_{+}^{n+1}\right) \cap$ $T_{2}^{2}\left(\mathbb{R}_{+}^{n+1}\right)$, then $f \in T_{2}^{p}\left(\mathbb{R}_{+}^{n+1}\right)$ for all $p \in[1,2]$, and hence, the decomposition (3.1) holds in $T_{2}^{p}\left(\mathbb{R}_{+}^{n+1}\right)$.

Proof. Observing that $\omega$ is of uniformly upper-type 1, we have

$$
\begin{aligned}
\int_{\mathbb{R}^{n}}[\mathcal{A}(f)(x)]^{p} d x \leq & \int_{\left\{x \in \mathbb{R}^{n}: \mathcal{A}(f)(x)<1\right\}} \mathcal{A}(f)(x) d x \\
& +\int_{\left\{x \in \mathbb{R}^{n}: \mathcal{A}(f)(x) \geq 1\right\}}[\mathcal{A}(f)(x)]^{2} d x
\end{aligned}
$$




$$
\begin{aligned}
& \lesssim \int_{\left\{x \in \mathbb{R}^{n}: \mathcal{A}(f)(x)<1\right\}} \omega(x, \mathcal{A}(f)(x)) d x+\|f\|_{T_{2}^{2}\left(\mathbb{R}_{+}^{n+1}\right)}^{2} \\
& <\infty
\end{aligned}
$$

which implies that $f \in T_{2}^{p}\left(\mathbb{R}_{+}^{n+1}\right)$. Then from Proposition 3.1, it follows that the decomposition (3.1) holds in $T_{2}^{p}\left(\mathbb{R}_{+}^{n+1}\right)$, which completes the proof of Corollary 3.1.

In what follows, let $T_{\omega}^{c}\left(\mathbb{R}_{+}^{n+1}\right)$ and $T_{2}^{p, c}\left(\mathbb{R}_{+}^{n+1}\right)$ denote the set of all functions in $T_{\omega}\left(\mathbb{R}_{+}^{n+1}\right)$ and $T_{2}^{p}\left(\mathbb{R}_{+}^{n+1}\right)$ with compact supports, respectively, where $p \in(0, \infty)$.

\section{LEMMA 3.2.}

(i) For all $p \in(0, \infty), T_{2}^{p, c}\left(\mathbb{R}_{+}^{n+1}\right) \subset T_{2}^{2, c}\left(\mathbb{R}_{+}^{n+1}\right)$. In particular, if $p \in(0,2]$, then $T_{2}^{p, c}\left(\mathbb{R}_{+}^{n+1}\right)$ coincides with $T_{2}^{2, c}\left(\mathbb{R}_{+}^{n+1}\right)$.

(ii) Let $\omega$ satisfy Assumption $(C)$. Then $T_{\omega}^{c}\left(\mathbb{R}_{+}^{n+1}\right)$ coincides with $T_{2}^{2, c}\left(\mathbb{R}_{+}^{n+1}\right)$.

Proof. By [13, (1.3), p. 306], we have $T_{2}^{p, c}\left(\mathbb{R}_{+}^{n+1}\right) \subset T_{2}^{2, c}\left(\mathbb{R}_{+}^{n+1}\right)$ for all $p \in(0, \infty)$. If $p \in(0,2]$, then from the Hölder inequality, it is easy to follow that $T_{2}^{2, c}\left(\mathbb{R}_{+}^{n+1}\right) \subset T_{2}^{p, c}\left(\mathbb{R}_{+}^{n+1}\right)$. Thus, (i) holds.

Let us prove (ii). To prove $T_{\omega}^{c}\left(\mathbb{R}_{+}^{n+1}\right) \subset T_{2}^{2, c}\left(\mathbb{R}_{+}^{n+1}\right)$, by (i), it suffices to show that $T_{\omega}^{c}\left(\mathbb{R}_{+}^{n+1}\right) \subset T_{2}^{p, c}\left(\mathbb{R}_{+}^{n+1}\right)$ for certain $p \in(0, \infty)$. Suppose that $f \in$ $T_{\omega}^{c}\left(\mathbb{R}_{+}^{n+1}\right)$ and that $\operatorname{supp} f \subset K$, where $K$ is a compact set in $\mathbb{R}_{+}^{n+1}$. Let $B$ be a ball in $\mathbb{R}^{n}$ such that $K \subset \widehat{B}$. Then $\operatorname{supp} \mathcal{A}(f) \subset B$. This, together with the uniformly lower-type property of $\omega$, yields that

$$
\begin{aligned}
\int_{\mathbb{R}^{n}}[\mathcal{A}(f)(x)]^{p_{\omega}} d x= & \int_{\left\{x \in \mathbb{R}^{n}: \mathcal{A}(f)(x)<1\right\}}[\mathcal{A}(f)(x)]^{p_{\omega}} d x \\
& +\int_{\left\{x \in \mathbb{R}^{n}: \mathcal{A}(f)(x) \geq 1\right\}}[\mathcal{A}(f)(x)]^{p_{\omega}} d x \\
\lesssim & |B|+\int_{\mathbb{R}^{n}} \omega(x, \mathcal{A}(f)(x)) d x<\infty .
\end{aligned}
$$

That is, $f \in T_{2}^{p_{\omega}, c}\left(\mathbb{R}_{+}^{n+1}\right) \subset T_{2}^{2, c}\left(\mathbb{R}_{+}^{n+1}\right)$.

Conversely, let $f \in T_{2}^{1, c}\left(\mathbb{R}_{+}^{n+1}\right)$ be supported in a compact set $K$ in $\mathbb{R}_{+}^{n+1}$. Then there exists a ball $B$ such that $K \subset \widehat{B}$ and $\operatorname{supp} \mathcal{A}(f) \subset B$. This, together with the uniformly upper-type property of $\omega$, implies that 


$$
\begin{aligned}
\int_{\mathbb{R}^{n}} \omega(x, \mathcal{A}(f)(x)) d x \lesssim & \int_{\left\{x \in \mathbb{R}^{n}: \mathcal{A}(f)(x)<1\right\}} \omega(x, 1) d x \\
& +\int_{\left\{x \in \mathbb{R}^{n}: \mathcal{A}(f)(x) \geq 1\right\}} \mathcal{A}(f)(x) d x \\
& \lesssim|B|+\|f\|_{T_{2}^{1}\left(\mathbb{R}_{+}^{n+1}\right)}<\infty
\end{aligned}
$$

from which it follows that $f \in T_{\omega}^{c}\left(\mathbb{R}_{+}^{n+1}\right)$, and hence, completes the proof of Lemma 3.2.

\section{§4. Musielak-Orlicz spaces and their dual spaces}

In this section, we always suppose that the Musielak-Orlicz function $\omega$ satisfies Assumption (C). We first introduce the Musielak-Orlicz Hardy space associated to $L$ via the Lusin area function and then establish its duality. Let us begin with some notions and notation.

Let $\mathcal{S}_{L}$ be the same as in (1.3). It follows from Lemma 2.4 that the operator $\mathcal{S}_{L}$ is bounded on $L^{p}\left(\mathbb{R}^{n}\right)$ for $p \in\left(p_{L}, \widetilde{p}_{L}\right)$. Hofmann and Mayboroda in [22] introduced the Hardy space $H_{L}^{1}\left(\mathbb{R}^{n}\right)$ associated to $L$ as the completion of $\left\{f \in L^{2}\left(\mathbb{R}^{n}\right): \mathcal{S}_{L} f \in L^{1}\left(\mathbb{R}^{n}\right)\right\}$ with respect to the norm $\|f\|_{H_{L}^{1}\left(\mathbb{R}^{n}\right)} \equiv\left\|\mathcal{S}_{L} f\right\|_{L^{1}\left(\mathbb{R}^{n}\right)}$.

Employing some ideas from [16], [22], and [24], we now introduce the Musielak-Orlicz Hardy space $H_{\omega, L}\left(\mathbb{R}^{n}\right)$ associated to $L$ and $\omega$ as follows.

Definition 4.1. Let $\omega$ satisfy Assumption (C). A function $f \in L^{2}\left(\mathbb{R}^{n}\right)$ is said to be in $\widetilde{H}_{\omega, L}\left(\mathbb{R}^{n}\right)$ if $\mathcal{S}_{L} f \in L(\omega)$; moreover, define

$$
\|f\|_{H_{\omega, L}\left(\mathbb{R}^{n}\right)} \equiv\left\|\mathcal{S}_{L} f\right\|_{L(\omega)}=\inf \left\{\lambda>0: \int_{\mathbb{R}^{n}} \omega\left(x, \frac{\mathcal{S}_{L} f(x)}{\lambda}\right) d x \leq 1\right\}
$$

The Musielak-Orlicz Hardy space $H_{\omega, L}\left(\mathbb{R}^{n}\right)$ is defined to be the completion of $\widetilde{H}_{\omega, L}\left(\mathbb{R}^{n}\right)$ in the norm $\|\cdot\|_{H_{\omega, L}\left(\mathbb{R}^{n}\right)}$.

In what follows, for a ball $B \equiv B\left(x_{B}, r_{B}\right)$, we let $U_{0}(B) \equiv B$, and for $j \in \mathbb{N}, U_{j}(B) \equiv B\left(x_{B}, 2^{j} r_{B}\right) \backslash B\left(x_{B}, 2^{j-1} r_{B}\right)$.

Definition 4.2. Let $q \in\left(p_{L}, \widetilde{p}_{L}\right)$, let $M \in \mathbb{N}$, and let $\epsilon \in(0, \infty)$. A function $\alpha \in L^{q}\left(\mathbb{R}^{n}\right)$ is called an $(\omega, q, M, \epsilon)$-molecule adapted to $B$ if there exists a ball $B$ such that

(i) $\|\alpha\|_{L^{q}\left(U_{j}(B)\right)} \lesssim 2^{-j \epsilon}\left|2^{j} B\right|^{\frac{1}{q}-1} \inf _{x \in B}\left[\rho\left(x,\left|2^{j} B\right|\right)\right]^{-1}, j \in \mathbb{Z}_{+}$; 
(ii) for every $k=1, \ldots, M$ and $j \in \mathbb{Z}_{+}$, there holds

$$
\left\|\left(r_{B}^{-2} L^{-1}\right)^{k} \alpha\right\|_{L^{q}\left(U_{j}(B)\right)} \lesssim 2^{-j \epsilon}\left|2^{j} B\right|^{\frac{1}{q}-1} \inf _{x \in B}\left[\rho\left(x,\left|2^{j} B\right|\right)\right]^{-1} .
$$

Finally, if $\alpha$ is an $(\omega, q, M, \epsilon)$-molecule for all $q \in\left(p_{L}, \widetilde{p}_{L}\right)$, then $\alpha$ is called an $(\omega, \infty, M, \epsilon)$-molecule.

REMARK 4.1 .

(i) Since $\omega$ is of strictly uniformly lower-type $p_{\omega}$, we have for all $f_{1}, f_{2} \in$ $H_{\omega, L}\left(\mathbb{R}^{n}\right)$,

$$
\left\|f_{1}+f_{2}\right\|_{H_{\omega, L}\left(\mathbb{R}^{n}\right)}^{p_{\omega}} \leq\left\|f_{1}\right\|_{H_{\omega, L}\left(\mathbb{R}^{n}\right)}^{p_{\omega}}+\left\|f_{2}\right\|_{H_{\omega, L}\left(\mathbb{R}^{n}\right)}^{p_{\omega}} .
$$

In fact, if letting $\lambda_{1} \equiv\left\|f_{1}\right\|_{H_{\omega, L}\left(\mathbb{R}^{n}\right)}^{p_{\omega}}$ and $\lambda_{2} \equiv\left\|f_{2}\right\|_{H_{\omega, L}\left(\mathbb{R}^{n}\right)}^{p_{\omega}}$, by the subadditivity, the continuity, and the uniformly lower-type $p_{\omega}$ of $\omega$, we have

$$
\begin{aligned}
\int_{\mathbb{R}^{n}} \omega\left(x, \frac{\mathcal{S}_{L}\left(f_{1}+f_{2}\right)(x)}{\left(\lambda_{1}+\lambda_{2}\right)^{\frac{1}{p_{\omega}}}}\right) d x & \leq \sum_{i=1}^{2} \int_{\mathbb{R}^{n}} \omega\left(x, \frac{\mathcal{S}_{L}\left(f_{i}\right)(x)}{\left(\lambda_{1}+\lambda_{2}\right)^{\frac{1}{p_{\omega}}}}\right) d x \\
& \leq \sum_{i=1}^{2} \frac{\lambda_{i}}{\lambda_{1}+\lambda_{2}} \int_{\mathbb{R}^{n}} \omega\left(x, \frac{\mathcal{S}_{L}\left(f_{i}\right)(x)}{\lambda_{i}^{\frac{1}{p_{\omega}}}}\right) d x \leq 1
\end{aligned}
$$

which implies that $\left\|f_{1}+f_{2}\right\|_{H_{\omega, L}\left(\mathbb{R}^{n}\right)} \leq\left(\left\|f_{1}\right\|_{H_{\omega, L}\left(\mathbb{R}^{n}\right)}^{p_{\omega}}+\left\|f_{2}\right\|_{H_{\omega, L}\left(\mathbb{R}^{n}\right)}^{p_{\omega}}\right)^{\frac{1}{p_{\omega}}}$, and hence, the desired conclusion.

(ii) From the theorem of completion of Yosida [37, p. 56], it follows that $\widetilde{H}_{\omega, L}\left(\mathbb{R}^{n}\right)$ is dense in $H_{\omega, L}\left(\mathbb{R}^{n}\right)$; namely, for any $f \in H_{\omega, L}\left(\mathbb{R}^{n}\right)$ there exists a Cauchy sequence $\left\{f_{k}\right\}_{k=1}^{\infty} \subset \widetilde{H}_{\omega, L}\left(\mathbb{R}^{n}\right)$ such that $\lim _{k \rightarrow \infty}\left\|f_{k}-f\right\|_{H_{\omega, L}\left(\mathbb{R}^{n}\right)}=0$. Moreover, if $\left\{f_{k}\right\}_{k=1}^{\infty}$ is a Cauchy sequence in $\widetilde{H}_{\omega, L}\left(\mathbb{R}^{n}\right)$, then there uniquely exists $f \in H_{\omega, L}\left(\mathbb{R}^{n}\right)$ such that $\lim _{k \rightarrow \infty}\left\|f_{k}-f\right\|_{H_{\omega, L}\left(\mathbb{R}^{n}\right)}=0$.

\subsection{Molecular decompositions of $H_{\omega, L}\left(\mathbb{R}^{n}\right)$}

In what follows, let $L_{c}^{2}\left(\mathbb{R}_{+}^{n+1}\right)$ denote the set of all functions in $L^{2}\left(\mathbb{R}_{+}^{n+1}\right)$ with compact supports.

Proposition 4.1. Let $\omega$ satisfy Assumption (C), let $M \in \mathbb{N}$ and $M>$ $\frac{n}{2}\left(\frac{1}{p_{\omega}}-\frac{1}{2}\right)$, and let $\pi_{L, M}$ be as in (1.4).

(i) The operator $\pi_{L, M}$, initially defined on $T_{2}^{p, c}\left(\mathbb{R}_{+}^{n+1}\right)$, extends to a bounded linear operator from $T_{2}^{p}\left(\mathbb{R}_{+}^{n+1}\right)$ to $L^{p}\left(\mathbb{R}^{n}\right)$, where $p \in\left(p_{L}, \widetilde{p}_{L}\right)$. 
(ii) The operator $\pi_{L, M}$, initially defined on $T_{\omega}^{c}\left(\mathbb{R}_{+}^{n+1}\right)$, extends to a bounded linear operator from $T_{\omega}\left(\mathbb{R}_{+}^{n+1}\right)$ to $H_{\omega, L}\left(\mathbb{R}^{n}\right)$.

Proof. Let $k \in \mathbb{N}$. By Lemma 2.4 and a duality argument, we know that the operator $\mathcal{S}_{L^{*}}^{k}$ is bounded on $L^{p}\left(\mathbb{R}^{n}\right)$ for $p \in\left(p_{L^{*}}, \widetilde{p}_{L^{*}}\right)$, where $\frac{1}{p_{L^{*}}}+\frac{1}{\widetilde{p}_{L}}=$ $1=\frac{1}{p_{L}}+\frac{1}{\tilde{p}_{L^{*}}}$. We refer the reader to [24, Proposition 4.1] for the proof of (i) above.

Let us prove (ii). Assume that $f \in T_{\omega}^{c}\left(\mathbb{R}_{+}^{n+1}\right)$. By Theorem 3.1, we have $f=\sum_{j=1}^{\infty} \lambda_{j} a_{j}$ pointwise, where $\left\{\lambda_{j}\right\}_{j=1}^{\infty}$ and $\left\{a_{j}\right\}_{j=1}^{\infty}$ are as in Theorem 3.1 and where $\Lambda\left(\left\{\lambda_{j} a_{j}\right\}_{j}\right) \lesssim\|f\|_{T_{\omega}\left(\mathbb{R}_{+}^{n+1}\right)}$. From Lemma 3.2(ii), it then follows that $f \in T_{2}^{2, c}\left(\mathbb{R}_{+}^{n+1}\right)$, which together with (i) and Corollary 3.1 further implies that

$$
\pi_{L, M}(f)=\sum_{j=1}^{\infty} \lambda_{j} \pi_{L, M}\left(a_{j}\right) \equiv \sum_{j=1}^{\infty} \lambda_{j} \alpha_{j}
$$

in $L^{p}\left(\mathbb{R}^{n}\right)$ for $p \in\left(p_{L}, 2\right]$.

On the other hand, notice that the operator $\mathcal{S}_{L}$ is bounded on $L^{p}\left(\mathbb{R}^{n}\right)$, which together with the subadditivity and the continuity of $\omega$ yields

$$
\int_{\mathbb{R}^{n}} \omega\left(x, \mathcal{S}_{L}\left(\pi_{L, M}(f)\right)(x)\right) d x \leq \sum_{j=1}^{\infty} \int_{\mathbb{R}^{n}} \omega\left(x,\left|\lambda_{j}\right| \mathcal{S}_{L}\left(\alpha_{j}\right)(x)\right) d x
$$

We next indicate that for any fixed $\epsilon \in(0, \infty), \alpha_{j}=\pi_{L, M}\left(a_{j}\right)$ is a multiple of an $(\omega, \infty, M, \epsilon)$-molecule adapted to $B_{j}$ for each $j$.

Indeed, this is the case provided that $a$ is an $(\omega, \infty)$-atom supported in the ball $B \equiv B\left(x_{B}, r_{B}\right)$ and that $q \in\left(p_{L}, \widetilde{p}_{L}\right)$. Since for $q \in\left(p_{L}, 2\right)$, each $(\omega, 2, M, \epsilon)$-molecule is also an $(\omega, q, M, \epsilon)$-molecule, to prove the above claim it suffices to show that $\alpha \equiv \pi_{L, M}(a)$ is a multiple of an $(\omega, q, M, \epsilon)$-molecule adapted to $B$ with $q \in\left[2, \widetilde{p}_{L}\right)$.

By (i), for $i=0,1,2$, we have

$$
\begin{aligned}
\|\alpha\|_{L^{q}\left(U_{i}(B)\right)} & =\left\|\pi_{L, M}(a)\right\|_{L^{q}\left(U_{i}(B)\right)} \lesssim\|a\|_{T_{2}^{q}\left(\mathbb{R}_{+}^{n+1}\right)} \\
& \lesssim|B|^{\frac{1}{q}-1} \inf _{x \in B}[\rho(x,|B|)]^{-1} .
\end{aligned}
$$

For $i \geq 3$, let $q^{\prime} \in(1,2]$ be the conjugate number of $q$, and let $h \in L^{q^{\prime}}\left(\mathbb{R}^{n}\right)$ satisfy $\|h\|_{L^{q^{\prime}\left(\mathbb{R}^{n}\right)}} \leq 1$ and $\operatorname{supp} h \subset U_{i}(B)$. By the Hölder inequality and Lemmas 2.1 and 2.3, we have 


$$
\begin{aligned}
\mid\langle & \left.\pi_{L, M}(a), h\right\rangle \mid \\
& \lesssim \int_{0}^{r_{B}} \int_{B}\left|a(x, t)\left(t^{2} L^{*}\right)^{M+1} e^{-t^{2} L^{*}}(h)(x)\right| \frac{d x d t}{t} \\
& \lesssim\|\mathcal{A}(a)\|_{L^{q}\left(\mathbb{R}^{n}\right)}|| \mathcal{A}\left(\chi_{\widehat{B}}\left(t^{2} L^{*}\right)^{M+1} e^{-t^{2} L^{*}}(h)\right) \|_{L^{q^{\prime}\left(\mathbb{R}^{n}\right)}} \\
\lesssim & \|a\|_{T_{2}^{q}\left(\mathbb{R}_{+}^{n+1}\right)}|B|^{\frac{1}{q^{\prime}}-\frac{1}{2}}\left(\int_{\widehat{B}}\left|\left(t^{2} L^{*}\right)^{M+1} e^{-t^{2} L^{*}}(h)(x, t)\right|^{2} \frac{d x d t}{t}\right)^{\frac{1}{2}} \\
\lesssim & \|a\|_{T_{2}^{q}\left(\mathbb{R}_{+}^{n+1}\right)}|B|^{\frac{1}{q^{\prime}}-\frac{1}{2}} \\
& \times\left(\int_{0}^{r_{B}}\left[t^{n\left(\frac{1}{2}-\frac{1}{q^{\prime}}\right)} \exp \left\{-\frac{\operatorname{dist}\left(B, U_{i}(B)\right)^{2}}{c t^{2}}\right\}\right]^{2} \frac{d t}{t}\right)^{\frac{1}{2}} \\
\lesssim & |B|^{-\frac{1}{2}} \inf _{x \in B}[\rho(x,|B|)]^{-1}\left(\int_{0}^{r_{B}} t^{n\left(1-\frac{2}{q^{\prime}}\right)}\left[\frac{t}{2^{i} r_{B}}\right]^{2\left(\epsilon+\frac{n}{p_{\omega}}-\frac{n}{q}\right)} \frac{d t}{t}\right)^{\frac{1}{2}} \\
& \lesssim 2^{-i \epsilon}\left|2^{i} B\right|^{\frac{1}{q}-1} \inf _{x \in B}\left[\rho\left(x,\left|2^{i} B\right|\right)\right]^{-1},
\end{aligned}
$$

which implies that $\alpha$ satisfies Definition 4.2(i).

We now show that $\alpha$ also satisfies Definition $4.2($ ii). Let $k \in\{1, \ldots, M\}$. If $i=0,1,2$, then let $h$ be the same as in the estimate of (4.2); similarly to the estimate of (4.1), we have

$$
\begin{aligned}
\left|\left\langle\left(r_{B}^{-2} L^{-1}\right)^{k} \pi_{L, M}(a), h\right\rangle\right| \\
\quad \lesssim \int_{0}^{r_{B}} \int_{B}\left(\frac{t}{r_{B}}\right)^{2 k}\left|a(x, t)\left(t^{2} L^{*}\right)^{M+1-k} e^{-t^{2} L^{*}}(h)(x)\right| \frac{d x d t}{t} \\
\quad \lesssim\|\mathcal{A}(a)\|_{L^{q}\left(\mathbb{R}^{n}\right)}\left\|\mathcal{S}_{L^{*}}^{M+1-k}(h)\right\|_{L^{q^{\prime}}\left(\mathbb{R}^{n}\right)} \\
\quad \lesssim\|a\|_{T_{2}^{q}\left(\mathbb{R}_{+}^{n+1}\right)} \lesssim|B|^{\frac{1}{q}-1} \inf _{x \in B}[\rho(x,|B|)]^{-1},
\end{aligned}
$$

which is the desired estimate, where we used the Hölder inequality and Lemma 2.4 by noting that $q^{\prime} \in\left(p_{L^{*}}, 2\right]$. If $i \geq 3$, an argument similar to that used in the estimate of (4.2) also yields the desired estimate. Thus, $\alpha=\pi_{L, M}(a)$ is a multiple of an $(\omega, q, M, \epsilon)$-molecule adapted to $B$ with $q \in\left[2, \widetilde{p}_{L}\right)$, and the claim is proved.

Let $q \in\left(p_{L}, \widetilde{p}_{L}\right)$, and let $\epsilon>n\left(\frac{1}{p_{\omega}}-\frac{1}{\tilde{p}_{\omega}}\right)$, where $\widetilde{p}_{\omega}$ is as in Convention $(\mathrm{B})$. We now assert that for all $(\omega, q, M, \epsilon)$-molecules $\alpha$ adapted to the ball $B \equiv$ $B\left(x_{B}, r_{B}\right)$ and $\lambda \in \mathbb{C}$,

$$
\int_{\mathbb{R}^{n}} \omega\left(x,|\lambda| \mathcal{S}_{L}(\alpha)(x)\right) d x \lesssim|B| \inf _{x \in \mathbb{R}^{n}} \omega\left(x, \frac{|\lambda|}{|B| \sup _{x \in B} \rho(x,|B|)}\right) .
$$


Once this is proved, then we deduce $\|\alpha\|_{H_{\omega, L}\left(\mathbb{R}^{n}\right)} \lesssim 1$, which together with (4.1) further implies that for all $f \in T_{\omega}^{c}\left(\mathbb{R}_{+}^{n+1}\right)$,

$$
\int_{\mathbb{R}^{n}} \omega\left(x, \mathcal{S}_{L}\left(\pi_{L, M}(f)\right)(x)\right) d x \lesssim \sum_{j=1}^{\infty}\left|B_{j}\right| \inf _{x \in \mathbb{R}^{n}} \omega\left(x, \frac{\left|\lambda_{j}\right|}{\left|B_{j}\right| \sup _{x \in B_{j}} \rho\left(x,\left|B_{j}\right|\right)}\right) .
$$

Thus, for all $f \in T_{\omega}^{c}\left(\mathbb{R}_{+}^{n+1}\right)$, we have

$$
\left\|\pi_{L, M}(f)\right\|_{H_{\omega, L}\left(\mathbb{R}^{n}\right)} \lesssim \Lambda\left(\left\{\lambda_{j} a_{j}\right\}_{j}\right) \lesssim\|f\|_{T_{\omega}\left(\mathbb{R}_{+}^{n+1}\right)},
$$

which if combined with a density argument implies (ii).

Now, let us prove assertion (4.3). Observe that if $q>2$, then an $(\omega, q, M, \epsilon)$ molecule is also an $(\omega, 2, M, \epsilon)$-molecule. Thus, to prove assertion (4.3), it suffices to show (4.3) for $q \in\left(p_{L}, 2\right]$. To this end, note that

$$
\begin{aligned}
\int_{\mathbb{R}^{n}} \omega & \left(x,|\lambda| \mathcal{S}_{L}(\alpha)(x)\right) d x \\
\leq & \int_{\mathbb{R}^{n}} \omega\left(x,|\lambda| \mathcal{S}_{L}\left(\left[I-e^{-r_{B}^{2} L}\right]^{M} \alpha\right)(x)\right) d x \\
& +\int_{\mathbb{R}^{n}} \omega\left(x,|\lambda| \mathcal{S}_{L}\left(\left(I-\left[I-e^{-r_{B}^{2} L}\right]^{M}\right) \alpha\right)(x)\right) d x \\
\lesssim & \sum_{j=0}^{\infty} \int_{\mathbb{R}^{n}} \omega\left(x,|\lambda| \mathcal{S}_{L}\left(\left[I-e^{-r_{B}^{2} L}\right]^{M}\left(\alpha \chi_{U_{j}(B)}\right)\right)(x)\right) d x \\
& +\sum_{j=0}^{\infty} \sup _{1 \leq k \leq M} \int_{\mathbb{R}^{n}} \omega\left(x,|\lambda| \mathcal{S}_{L}\left\{\left[\frac{k}{M} r_{B}^{2} L e^{\left.-\frac{k}{M} r_{B}^{2} L\right]^{M}}\right.\right.\right. \\
& \left.\left.\times\left(\chi_{U_{j}(B)}\left(r_{B}^{-2} L^{-1}\right)^{M} \alpha\right)\right\}(x)\right) d x \\
\equiv & \sum_{j=0}^{\infty} \mathrm{H}_{j}+\sum_{j=0}^{\infty} \mathrm{I}_{j} .
\end{aligned}
$$

For each $j \geq 0$, let $B_{j} \equiv 2^{j} B$. Then, by Assumption (C) and the Hölder inequality, we obtain

$$
\begin{aligned}
\mathrm{H}_{j} & \lesssim \sum_{k=0}^{\infty} \int_{U_{k}\left(B_{j}\right)} \omega\left(x,|\lambda| \mathcal{S}_{L}\left(\left[I-e^{-r_{B}^{2} L}\right]^{M}\left(\alpha \chi_{U_{j}(B)}\right)\right)(x)\right) d x \\
& \lesssim \sum_{k=0}^{\infty} \int_{2^{k} B_{j}} \omega\left(x,|\lambda| \chi_{U_{k}\left(B_{j}\right)}(x) \mathcal{S}_{L}\left(\left[I-e^{-r_{B}^{2} L}\right]^{M}\left(\alpha \chi_{U_{j}(B)}\right)\right)(x)\right) d x
\end{aligned}
$$




$$
\begin{aligned}
& \lesssim \sum_{k=0}^{\infty}\left|2^{k} B_{j}\right| \inf _{x \in \mathbb{R}^{n}} \omega\left(x, \frac{|\lambda|}{\left|2^{k} B_{j}\right|} \int_{U_{k}\left(B_{j}\right)} \mathcal{S}_{L}\left(\left[I-e^{-r_{B}^{2} L}\right]^{M}\left(\alpha \chi_{U_{j}(B)}\right)\right)(x) d x\right) \\
& \lesssim \sum_{k=0}^{\infty}\left|2^{k} B_{j}\right| \\
& \quad \times \inf _{x \in \mathbb{R}^{n}} \omega\left(x, \frac{|\lambda|}{\left|2^{k} B_{j}\right|^{1 / q}}\left\|\mathcal{S}_{L}\left(\left[I-e^{-r_{B}^{2} L}\right]^{M}\left(\alpha \chi_{U_{j}(B)}\right)\right)\right\|_{L^{q}\left(U_{k}\left(B_{j}\right)\right)}\right) .
\end{aligned}
$$

By the proof of $[22$, Lemma 4.2] (see [22, (4.22) and (4.27)]), we have for $k=0,1,2$,

$$
\left\|\mathcal{S}_{L}\left(\left[I-e^{-r_{B}^{2} L}\right]^{M}\left(\alpha \chi_{U_{j}(B)}\right)\right)\right\|_{L^{q}\left(U_{k}\left(B_{j}\right)\right)} \lesssim\|\alpha\|_{L^{q}\left(U_{j}(B)\right)},
$$

and for $k \geq 3$,

$$
\begin{gathered}
\left\|\mathcal{S}_{L}\left(\left[I-e^{-r_{B}^{2} L}\right]^{M}\left(\alpha \chi_{U_{j}(B)}\right)\right)\right\|_{L^{q}\left(U_{k}\left(B_{j}\right)\right)}^{2} \\
\lesssim k\left(\frac{1}{2^{k+j}}\right)^{4 M+2\left(\frac{n}{2}-\frac{n}{q}\right)}\|\alpha\|_{L^{q}\left(U_{j}(B)\right)}^{2},
\end{gathered}
$$

which, together with Definition 4.2, $2 M p_{\omega}>n\left(1-\frac{p_{\omega}}{2}\right)$, and Assumption (C), implies that

$$
\begin{aligned}
\mathrm{H}_{j} \lesssim & \left|B_{j}\right| \inf _{x \in \mathbb{R}^{n}} \omega\left(x, \frac{|\lambda| 2^{-j \epsilon}}{\left|B_{j}\right| \sup _{x \in B_{j}} \rho\left(x,\left|B_{j}\right|\right)}\right) \\
& +\sum_{k=3}^{\infty}\left|2^{k} B_{j}\right| \inf _{x \in \mathbb{R}^{n}} \omega\left(x, \frac{|\lambda| \sqrt{k} 2^{-\left(2 M+\frac{n}{2}-\frac{n}{q}\right)(j+k)-j \epsilon}}{\left|2^{k} B_{j}\right|^{\frac{1}{q}}\left|B_{j}\right|^{1-\frac{1}{q}} \sup _{x \in B_{j}} \rho\left(x,\left|B_{j}\right|\right)}\right) \\
\lesssim & 2^{-j p_{\omega} \epsilon}\left\{1+\sum_{k=3}^{\infty} \sqrt{k} 2^{k n\left(1-\frac{p_{\omega}}{q}\right)} 2^{-p_{\omega}\left(2 M+\frac{n}{2}-\frac{n}{q}\right)(j+k)}\right\} \\
& \times\left|B_{j}\right| \inf _{x \in \mathbb{R}^{n}} \omega\left(x, \frac{|\lambda|}{\left|B_{j}\right| \sup _{x \in B_{j}} \rho\left(x,\left|B_{j}\right|\right)}\right) \\
\lesssim & 2^{-j p_{\omega} \epsilon}\left|B_{j}\right| \inf _{x \in \mathbb{R}^{n}} \omega\left(x, \frac{|\lambda|}{\left|B_{j}\right| \sup _{x \in B_{j}} \rho\left(x,\left|B_{j}\right|\right)}\right) .
\end{aligned}
$$

Note that since $\epsilon>n\left(\frac{1}{p_{\omega}}-\frac{1}{\widetilde{p}_{\omega}}\right)$ and $\omega^{-1}$ is of uniformly lower-type $\frac{1}{\widetilde{p}_{\omega}}$ by Lemma 2.6, we further have 


$$
\begin{aligned}
\sum_{j=0}^{\infty} \mathrm{H}_{j} \lesssim & \sum_{j=0}^{\infty} 2^{-j p_{\omega} \epsilon}\left|B_{j}\right|\left\{\frac{|B| \sup _{x \in B} \rho(x,|B|)}{\left|B_{j}\right| \sup _{x \in B_{j}} \rho\left(x,\left|B_{j}\right|\right)}\right\}^{p_{\omega}} \\
& \times \inf _{x \in \mathbb{R}^{n}} \omega\left(x, \frac{|\lambda|}{|B| \sup _{x \in B} \rho(x,|B|)}\right) \\
\lesssim & \sum_{j=0}^{\infty} 2^{-j p_{\omega} \epsilon}\left|B_{j}\right|\left\{\frac{|B|}{\left|B_{j}\right|}\right\}^{\frac{p_{\omega}}{\tilde{p}}} \inf _{x \in \mathbb{R}^{n}} \omega\left(x, \frac{|\lambda|}{|B| \sup _{x \in B} \rho(x,|B|)}\right) \\
\lesssim & \sum_{j=0}^{\infty} 2^{-j p_{\omega} \epsilon} 2^{j n\left(1-\frac{p_{\omega}}{\tilde{p}_{\omega}}\right)}|B| \inf _{x \in \mathbb{R}^{n}} \omega\left(x, \frac{|\lambda|}{|B| \sup _{x \in B} \rho(x,|B|)}\right) \\
\lesssim & |B| \inf _{x \in \mathbb{R}^{n}} \omega\left(x, \frac{|\lambda|}{|B| \sup _{x \in B} \rho(x,|B|)}\right) .
\end{aligned}
$$

Similarly, we have

$$
\sum_{j=0}^{\infty} \mathrm{I}_{j} \lesssim|B| \inf _{x \in \mathbb{R}^{n}} \omega\left(x, \frac{|\lambda|}{|B| \sup _{x \in B} \rho(x,|B|)}\right)
$$

which completes the estimate of (4.3), and hence, the proof of Proposition 4.1 .

Proposition 4.2. Let $\omega$ satisfy Assumption (C), let $\epsilon>n\left(\frac{1}{p_{\omega}}-\frac{1}{p_{\omega}^{+}}\right)$, and let $M>\frac{n}{2}\left(\frac{1}{p_{\omega}}-\frac{1}{2}\right)$. If $f \in H_{\omega, L}\left(\mathbb{R}^{n}\right) \cap L^{2}\left(\mathbb{R}^{n}\right)$, then $f \in L^{p}\left(\mathbb{R}^{n}\right)$ for all $p \in\left(p_{L}, 2\right]$ and there exist $(\omega, \infty, M, \epsilon)$-molecules $\left\{\alpha_{j}\right\}_{j=1}^{\infty}$ and numbers $\left\{\lambda_{j}\right\}_{j=1}^{\infty} \subset \mathbb{C}$ such that

$$
f=\sum_{j=1}^{\infty} \lambda_{j} \alpha_{j}
$$

in both $H_{\omega, L}\left(\mathbb{R}^{n}\right)$ and $L^{p}\left(\mathbb{R}^{n}\right)$ for all $p \in\left(p_{L}, 2\right]$. Moreover, there exists a positive constant $C$ independent of $f$ such that for all $f \in H_{\omega, L}\left(\mathbb{R}^{n}\right) \cap L^{2}\left(\mathbb{R}^{n}\right)$,

$$
\begin{aligned}
\Lambda\left(\left\{\lambda_{j} \alpha_{j}\right\}_{j}\right) & \equiv \inf \left\{\lambda>0: \sum_{j=1}^{\infty}\left|B_{j}\right| \inf _{x \in \mathbb{R}^{n}} \omega\left(x, \frac{\left|\lambda_{j}\right|}{\lambda\left|B_{j}\right| \sup _{x \in B_{j}} \rho\left(x,\left|B_{j}\right|\right)}\right) \leq 1\right\} \\
& \leq C\|f\|_{H_{\omega, L}\left(\mathbb{R}^{n}\right)},
\end{aligned}
$$

where for each $j, \alpha_{j}$ is adapted to the ball $B_{j}$. 
Proof. Let $f \in H_{\omega, L}\left(\mathbb{R}^{n}\right) \cap L^{2}\left(\mathbb{R}^{n}\right)$. For each $N \in \mathbb{N}$, define $O_{N} \equiv\{(x, t) \in$ $\left.\mathbb{R}_{+}^{n+1}:|x|<N, 1 / N<t<N\right\}$. Then by the $L^{2}\left(\mathbb{R}^{n}\right)$ functional calculi for $L$, we have

$$
f=C_{M} \int_{0}^{\infty}\left(t^{2} L\right)^{M+2} e^{-2 t^{2} L} f \frac{d t}{t}=\lim _{N \rightarrow \infty} \pi_{L, M}\left(\chi_{O_{N}}\left(t^{2} L e^{-t^{2} L} f\right)\right)
$$

in $L^{2}\left(\mathbb{R}^{n}\right)$, where $M \in \mathbb{N}, \pi_{L, M}$ and $C_{M}$ are as in (1.4).

On the other hand, by Definition 4.1 and Lemma 2.4, we have $t^{2} L e^{-t^{2} L} f \in T_{2}^{2}\left(\mathbb{R}_{+}^{n+1}\right) \cap T_{\omega}\left(\mathbb{R}_{+}^{n+1}\right)$. An application of Corollary 3.1 shows that $t^{2} L e^{-t^{2} L} f \in T_{2}^{p}\left(\mathbb{R}_{+}^{n+1}\right)$, which together with Proposition 4.1(i) implies that $\left\{\pi_{L, M}\left(\chi_{O_{N}}\left(t^{2} L e^{-t^{2} L} f\right)\right)\right\}_{N}$ is a Cauchy sequence in $L^{p}\left(\mathbb{R}^{n}\right)$. Then by taking subsequence, we have

$$
f=\lim _{N \rightarrow \infty} \pi_{L, M}\left(\chi_{O_{N}}\left(t^{2} L e^{-t^{2} L} f\right)\right)
$$

in $L^{p}\left(\mathbb{R}^{n}\right)$.

Now applying Theorem 3.1 and Proposition 3.1 to $t^{2} L e^{-t^{2} L} f$, we obtain $(\omega, \infty)$-atoms $\left\{a_{j}\right\}_{j=1}^{\infty}$ and numbers $\left\{\lambda_{j}\right\}_{j=1}^{\infty} \subset \mathbb{C}$ such that $t^{2} L e^{-t^{2} L} f=$ $\sum_{j=1}^{\infty} \lambda_{j} a_{j}$ in $T_{2}^{p}\left(\mathbb{R}_{+}^{n+1}\right)$ and $\Lambda\left(\left\{\lambda_{j} a_{j}\right\}_{j}\right) \lesssim\left\|t^{2} L e^{-t^{2} L} f\right\|_{T_{\omega}\left(\mathbb{R}_{+}^{n+1}\right)}$, which, combined with Proposition 4.1(i), further yield that

$$
f=\pi_{L, M}\left(t^{2} L e^{-t^{2} L} f\right)=\sum_{j=1}^{\infty} \lambda_{j} \pi_{L, M}\left(a_{j}\right) \equiv \sum_{j=1}^{\infty} \lambda_{j} \alpha_{j}
$$

in $L^{p}\left(\mathbb{R}^{n}\right)$ for $p \in\left(p_{L}, 2\right]$. By the proof of Proposition 4.1, we learn that $\alpha_{j}$ is a multiple of an $(\omega, \infty, M, \epsilon)$-molecule for any $\epsilon>0$, and $M \in \mathbb{N}$ and $M>$ $\frac{n}{2}\left(\frac{1}{p_{\omega}}-\frac{1}{2}\right)$. Note that $\Lambda\left(\left\{\lambda_{j} \alpha_{j}\right\}_{j}\right)=\Lambda\left(\left\{\lambda_{j} a_{j}\right\}_{j}\right)$. We therefore obtain (4.6).

To finish the proof of Proposition 4.2, it remains to show that (4.5) holds in $H_{\omega, L}\left(\mathbb{R}^{n}\right)$. In fact, by Lemma 2.4, (3.3), (4.3), and (4.7) together with the continuity and the subadditivity of $\omega$, we have

$$
\begin{aligned}
& \int_{\mathbb{R}^{n}} \omega\left(x, \mathcal{S}_{L}\left(f-\sum_{j=1}^{N} \lambda_{j} \alpha_{j}\right)(x)\right) d x \\
& \quad \leq \sum_{j=N+1}^{\infty} \int_{\mathbb{R}^{n}} \omega\left(x, \mathcal{S}_{L}\left(\lambda_{j} \alpha_{j}\right)(x)\right) d x \\
& \quad \lesssim \sum_{j=N+1}^{\infty}\left|B_{j}\right| \inf _{x \in \mathbb{R}^{n}} \omega\left(x, \frac{\left|\lambda_{j}\right|}{\left|B_{j}\right| \sup _{x \in B_{j}}\left|\rho\left(x,\left|B_{j}\right|\right)\right|}\right) \rightarrow 0
\end{aligned}
$$


as $N \rightarrow \infty$. It should be pointed out that here, in the last inequality, to use (4.3), we need to choose $\widetilde{p}_{\omega}$ as in Convention (C) such that $\epsilon>n\left(\frac{1}{p_{\omega}}-\right.$ $\left.\frac{1}{\widetilde{p}_{\omega}}\right)$, which is guaranteed by the assumption $\epsilon>n\left(\frac{1}{p_{\omega}}-\frac{1}{p_{\omega}^{+}}\right)$. This, together with an argument similar to the proof of Proposition 3.1, yields that $f=$ $\sum_{j=1}^{\infty} \lambda_{j} \alpha_{j}$ in $H_{\omega, L}\left(\mathbb{R}^{n}\right)$, which completes the proof of Proposition 4.2.

Corollary 4.1. Let $\omega$ satisfy Assumption $(C)$, let $\epsilon>n\left(\frac{1}{p_{\omega}}-\frac{1}{p_{\omega}^{+}}\right)$, let $q \in\left(p_{L}, \widetilde{p}_{L}\right)$, and let $M>\frac{n}{2}\left(\frac{1}{p_{\omega}}-\frac{1}{2}\right)$. Then for every $f \in H_{\omega, L}\left(\mathbb{R}^{n}\right)$, there exist $(\omega, q, M, \epsilon)$-molecules $\left\{\alpha_{j}\right\}_{j=1}^{\infty}$ and numbers $\left\{\lambda_{j}\right\}_{j=1}^{\infty} \subset \mathbb{C}$ such that $f=$ $\sum_{j=1}^{\infty} \lambda_{j} \alpha_{j}$ in $H_{\omega, L}\left(\mathbb{R}^{n}\right)$. Furthermore, if letting $\Lambda\left(\left\{\lambda_{j} \alpha_{j}\right\}_{j}\right)$ be as in (4.6), then there exists a positive constant $C$ independent of $f$ such that $\Lambda\left(\left\{\lambda_{j} \alpha_{j}\right\}_{j}\right) \leq C\|f\|_{H_{\omega, L}\left(\mathbb{R}^{n}\right)}$.

Proof. If $f \in H_{\omega, L}\left(\mathbb{R}^{n}\right) \cap L^{2}\left(\mathbb{R}^{n}\right)$, then it immediately follows from Proposition 4.2 that all results hold.

Otherwise, there exist $\left\{f_{k}\right\}_{k=1}^{\infty} \subset\left(H_{\omega, L}\left(\mathbb{R}^{n}\right) \cap L^{2}\left(\mathbb{R}^{n}\right)\right)$ such that for all $k \in \mathbb{N}$,

$$
\left\|f-f_{k}\right\|_{H_{\omega, L}\left(\mathbb{R}^{n}\right)} \leq 2^{-k}\|f\|_{H_{\omega, L}\left(\mathbb{R}^{n}\right)} .
$$

Set $f_{0} \equiv 0$. Then $f=\sum_{k=1}^{\infty}\left(f_{k}-f_{k-1}\right)$ in $H_{\omega, L}\left(\mathbb{R}^{n}\right)$. By Proposition 4.2, we know that for all $k \in \mathbb{N}, f_{k}-f_{k-1}=\sum_{j=1}^{\infty} \lambda_{j}^{k} \alpha_{j}^{k}$ in $H_{\omega, L}\left(\mathbb{R}^{n}\right)$ and $\Lambda\left(\left\{\lambda_{j}^{k} a_{j}^{k}\right\}_{j}\right) \lesssim\left\|f_{k}-f_{k-1}\right\|_{H_{\omega, L}\left(\mathbb{R}^{n}\right)}$, where for all $j$ and $k, \alpha_{j}^{k}$ is an $(\omega, q, M, \epsilon)$ molecule. Thus, $f=\sum_{k, j=1}^{\infty} \lambda_{j}^{k} \alpha_{j}^{k}$ in $H_{\omega, L}\left(\mathbb{R}^{n}\right)$, and it further follows from Remark 3.2 that

$$
\begin{aligned}
{\left[\Lambda\left(\left\{\lambda_{j}^{k} \alpha_{j}^{k}\right\}_{k, j}\right)\right]^{p_{\omega}} } & \leq \sum_{k=1}^{\infty}\left[\Lambda\left(\left\{\lambda_{j}^{k} a_{j}^{k}\right\}_{j}\right)\right]^{p_{\omega}} \lesssim \sum_{k=1}^{\infty}\left\|f_{k}-f_{k-1}\right\|_{H_{\omega, L}\left(\mathbb{R}^{n}\right)}^{p_{\omega}} \\
& \lesssim\|f\|_{H_{\omega, L}\left(\mathbb{R}^{n}\right)}^{p_{\omega}}
\end{aligned}
$$

which completes the proof of Corollary 4.1.

Let $H_{\omega, \mathrm{fin}}^{q, \epsilon}\left(\mathbb{R}^{n}\right)$ denote the set of all finite combinations of $(\omega, q, M, \epsilon)$ molecules. From Corollary 4.1, we immediately deduce the following density result.

Corollary 4.2. Let $\omega$ satisfy Assumption $(C)$, let $\epsilon>n\left(\frac{1}{p_{\omega}}-\frac{1}{p_{\omega}^{+}}\right)$, and let $M>\frac{n}{2}\left(\frac{1}{p_{\omega}}-\frac{1}{2}\right)$. Then the space $H_{\omega, \mathrm{fin}}^{q, M, \epsilon}\left(\mathbb{R}^{n}\right)$ is dense in the space $H_{\omega, L}\left(\mathbb{R}^{n}\right)$. 


\subsection{Dual spaces of $H_{\omega, L}\left(\mathbb{R}^{n}\right)$}

In this section, we study the dual space of the Musielak-Orlicz Hardy space $H_{\omega, L}\left(\mathbb{R}^{n}\right)$. We begin with some notions.

Following [22], for $\epsilon>0$ and $M \in \mathbb{N}$, we introduce the space

$$
\mathcal{M}_{\omega}^{M, \epsilon}(L) \equiv\left\{\mu \in L^{2}\left(\mathbb{R}^{n}\right):\|\mu\|_{\mathcal{M}_{\omega}^{M, \epsilon}(L)}<\infty\right\}
$$

where

$$
\begin{aligned}
\|\mu\|_{\mathcal{M}_{\omega}^{M, \epsilon}(L)} \equiv & \sup _{j \geq 0}\left\{2^{j \epsilon}\left|B\left(0,2^{j}\right)\right|^{1 / 2} \sup _{x \in B\left(0,2^{j}\right)} \rho\left(x,\left|B\left(0,2^{j}\right)\right|\right)\right. \\
& \left.\times \sum_{k=0}^{M}\left\|L^{-k} \mu\right\|_{L^{2}\left(U_{j}(B(0,1))\right)}\right\} .
\end{aligned}
$$

Note that if $\phi \in \mathcal{M}_{\omega}^{M, \epsilon}(L)$ with norm 1 , then $\phi$ is an $(\omega, 2, M, \epsilon)$-molecule adapted to $B(0,1)$. Conversely, if $\alpha$ is an $(\omega, 2, M, \epsilon)$-molecule adapted to a certain ball, then $\alpha \in \mathcal{M}_{\omega}^{M, \epsilon}(L)$.

Let $A_{t}$ denote either $\left(I+t^{2} L\right)^{-1}$ or $e^{-t^{2} L}$, and let $f \in\left(\mathcal{M}_{\omega}^{M, \epsilon}(L)\right)^{*}$, the dual of $\mathcal{M}_{\omega}^{M, \epsilon}(L)$. We claim that $\left(I-A_{t}^{*}\right)^{M} f \in L_{\text {loc }}^{2}\left(\mathbb{R}^{n}\right)$ in the sense of distributions. In fact, for any ball $B$, if $\psi \in L^{2}(B)$, then it follows from the Gaffney estimates via Lemmas 2.1 and 2.2 that $\left(I-A_{t}\right)^{M} \psi \in \mathcal{M}_{\omega}^{M, \epsilon}(L)$ for all $\epsilon>0$ and any fixed $t \in(0, \infty)$. Thus,

$$
\begin{aligned}
\left|\left\langle\left(I-A_{t}^{*}\right)^{M} f, \psi\right\rangle\right| & \equiv\left|\left\langle f,\left(I-A_{t}\right)^{M} \psi\right\rangle\right| \\
& \leq C\left(t, r_{B}, \operatorname{dist}(B, 0)\right)\|f\|_{\left(\mathcal{M}_{\omega}^{M, \epsilon}(L)\right)^{*}}\|\psi\|_{L^{2}(B)},
\end{aligned}
$$

which implies that $\left(I-A_{t}^{*}\right)^{M} f \in L_{\text {loc }}^{2}\left(\mathbb{R}^{n}\right)$ in the sense of distributions.

Finally, for any $M \in \mathbb{N}$, define

$$
\mathcal{M}_{\omega, L^{*}}^{M}\left(\mathbb{R}^{n}\right) \equiv \bigcap_{\epsilon>n\left(\frac{1}{p_{\omega}}-\frac{1}{p_{\omega}^{+}}\right)}\left(\mathcal{M}_{\omega}^{M, \epsilon}(L)\right)^{*}
$$

Definition 4.3. Let $q \in\left(p_{L}, \widetilde{p}_{L}\right)$, let $\omega$ satisfy Assumption $(\mathrm{C})$, and let $M>\frac{n}{2}\left(\frac{1}{p_{\omega}}-\frac{1}{2}\right)$. A functional $f \in \mathcal{M}_{\omega, L}^{M}\left(\mathbb{R}^{n}\right)$ is said to be in $\mathrm{BMO}_{\rho, L}^{q, M}\left(\mathbb{R}^{n}\right)$ if

$$
\begin{aligned}
\|f\|_{\mathrm{BMO}_{\rho, L}^{q, M}\left(\mathbb{R}^{n}\right)} & \equiv \sup _{B \subset \mathbb{R}^{n}} \frac{1}{\sup _{x \in B} \rho(x,|B|)}\left[\frac{1}{|B|} \int_{B}\left|\left(I-e^{-r_{B}^{2} L}\right)^{M} f(x)\right|^{q} d x\right]^{\frac{1}{q}} \\
& <\infty
\end{aligned}
$$

where the supremum is taken over all balls $B$ in $\mathbb{R}^{n}$. 
In what follows, when $q=2$, we denote $\mathrm{BMO}_{\rho, L}^{q, M}\left(\mathbb{R}^{n}\right)$ simply by $\operatorname{BMO}_{\rho, L}^{M}\left(\mathbb{R}^{n}\right)$. The proofs of Lemmas 4.1, 4.2, and 4.3 below are similar to those of Lemmas 8.1 and 8.3 of [22] and Lemma 4.3 of [24], respectively, and hence we skip them here.

Lemma 4.1. Let $\omega, q$, and $M$ be as in Definition 4.3. A functional $f \in$ $\mathrm{BMO}_{\rho, L}^{q, M}\left(\mathbb{R}^{n}\right)$ if and only if $f \in \mathcal{M}_{\omega, L}^{M}\left(\mathbb{R}^{n}\right)$ and

$$
\sup _{B \subset \mathbb{R}^{n}} \frac{1}{\sup _{x \in B} \rho(x,|B|)}\left[\frac{1}{|B|} \int_{B}\left|\left(I-\left(I+r_{B}^{2} L\right)^{-1}\right)^{M} f(x)\right|^{q} d x\right]^{\frac{1}{q}}<\infty .
$$

Moreover, the quantity appearing on the left-hand side of the above formula is equivalent to $\|f\|_{\mathrm{BMO}_{\rho, L}^{q, M}\left(\mathbb{R}^{n}\right)}$.

Lemma 4.2. Let $\omega$ and $M$ be as in Definition 4.3. Then there exists a positive constant $C$ such that, for all $f \in \mathrm{BMO}_{\rho, L}^{M}\left(\mathbb{R}^{n}\right)$,

$$
\begin{aligned}
& \sup _{B \subset \mathbb{R}^{n}} \frac{1}{\sup _{x \in B} \rho(x,|B|)}\left[\frac{1}{|B|} \iint_{\widehat{B}}\left|\left(t^{2} L\right)^{M} e^{-t^{2} L} f(x)\right|^{2} \frac{d x d t}{t}\right]^{1 / 2} \\
& \quad \leq C\|f\|_{\mathrm{BMO}_{\rho, L}^{M}\left(\mathbb{R}^{n}\right)}
\end{aligned}
$$

Lemma 4.3. Let $\omega, \rho$, and $M$ be as in Definition 4.3, let $q \in\left(p_{L^{*}}, 2\right]$, let $\epsilon, \epsilon_{1}>0$, and let $\widetilde{M}>M+\epsilon_{1}+\frac{n}{4}$. Suppose that $f \in \mathcal{M}_{\omega, L^{*}}^{M}\left(\mathbb{R}^{n}\right)$ satisfies

$$
\int_{\mathbb{R}^{n}} \frac{\left|\left(I-\left(I+L^{*}\right)^{-1}\right)^{M} f(x)\right|^{q}}{1+|x|^{n+\epsilon_{1}}} d x<\infty .
$$

Then for every $\left(\omega, q^{\prime}, \widetilde{M}, \epsilon\right)$-molecule $\alpha$,

$$
\langle f, \alpha\rangle=\widetilde{C}_{M} \iint_{\mathbb{R}_{+}^{n+1}}\left(t^{2} L^{*}\right)^{M} e^{-t^{2} L^{*}} f(x) \overline{t^{2} L e^{-t^{2} L} \alpha(x)} \frac{d x d t}{t},
$$

where $q^{\prime} \in[2, \infty)$ satisfying $\frac{1}{q}+\frac{1}{q^{\prime}}=1$ and $\widetilde{C}_{M}$ is a positive constant satisfying

$$
\widetilde{C}_{M} \int_{0}^{\infty} t^{2(M+1)} e^{-2 t^{2}} \frac{d t}{t}=1
$$

From Lemma 4.1, it is easy to follow that all $f \in \mathrm{BMO}_{\rho, L}^{q, M}\left(\mathbb{R}^{n}\right)$ satisfy (4.8) for all $\epsilon_{1} \in(0, \infty)$, and hence, Lemma 4.3 holds for all $f \in \mathrm{BMO}_{\rho, L}^{q, M}\left(\mathbb{R}^{n}\right)$.

Now, let us give the main results of this section. 
TheOrem 4.1. Let $\omega$ satisfy Assumption $(C)$, let $\epsilon>n\left(\frac{1}{p_{\omega}}-\frac{1}{p_{\omega}^{+}}\right)$, let $M>\frac{n}{2}\left(\frac{1}{p_{\omega}}-\frac{1}{2}\right)$, and let $\widetilde{M}>M+\frac{n}{4}$. In addition, assume that there exists a positive constant $K$ such that, for all balls $B$ in $\mathbb{R}^{n}$, for all $x \in \mathbb{R}^{n}$,

$$
|B| \omega\left[x, K \inf _{x \in B} \omega^{-1}\left(x,|B|^{-1}\right)\right] \geq 1 .
$$

Then $\left(H_{\omega, L}\left(\mathbb{R}^{n}\right)\right)^{*}$, the dual space of $H_{\omega, L}\left(\mathbb{R}^{n}\right)$, coincides with $\mathrm{BMO}_{\rho, L^{*}}^{M}\left(\mathbb{R}^{n}\right)$ in the following sense.

(i) Let $g \in \mathrm{BMO}_{\rho, L^{*}}^{M}\left(\mathbb{R}^{n}\right)$. Then the linear functional $\ell$, which is initially defined on $\left.H_{\omega, \mathrm{in}}^{2, \widetilde{M}}, \mathbb{R}^{n}\right)$ by

$$
\ell(f) \equiv\langle g, f\rangle
$$

has a unique extension to $H_{\omega, L}\left(\mathbb{R}^{n}\right)$ with $\|\ell\|_{\left(H_{\omega, L}\left(\mathbb{R}^{n}\right)\right)^{*}} \leq C \times$ $\|g\|_{\mathrm{BMO}_{\rho, L^{*}}^{M}\left(\mathbb{R}^{n}\right)}$, where $C$ is a positive constant independent of $g$.

(ii) Conversely, for any $\ell \in\left(H_{\omega, L}\left(\mathbb{R}^{n}\right)\right)^{*}$, then $\ell \in \mathrm{BMO}_{\rho, L^{*}}^{M}\left(\mathbb{R}^{n}\right)$, (4.10) holds for all $f \in H_{\omega, \text { fin }}^{2, M, \epsilon}\left(\mathbb{R}^{n}\right)$, and $\|\ell\|_{\mathrm{BMO}_{\rho, L^{*}}^{M}\left(\mathbb{R}^{n}\right)} \leq C\|\ell\|_{\left(H_{\omega, L}\left(\mathbb{R}^{n}\right)\right)^{*}}$, where $C$ is a positive constant independent of $\ell$.

Proof. Before coming to the proof of Theorem 4.1, we need the following lemma.

LEMMA 4.4. Let $\omega$ satisfy the assumptions of Theorem 4.1, and let $\left\{\lambda_{j}\right\}_{j=1}^{\infty} \subset \mathbb{C}$ and $\Lambda\left(\left\{\lambda_{j} a_{j}\right\}_{j}\right)$ be as in Theorem 3.1. Then we have

$$
\sum_{j=1}^{\infty}\left|\lambda_{j}\right| \leq C \Lambda\left(\left\{\lambda_{j} a_{j}\right\}_{j}\right) \leq C\|f\|_{T_{\omega}\left(\mathbb{R}_{+}^{n+1}\right)}
$$

Proof of Lemma 4.4. Take any $\lambda>0$ such that

$$
\sum_{j=1}^{\infty}\left|B_{j}\right| \inf _{x \in \mathbb{R}^{n}} \omega\left(x, \frac{\left|\lambda_{j}\right|}{\lambda\left|B_{j}\right| \sup _{x \in B_{j}} \rho\left(x,\left|B_{j}\right|\right)}\right) \leq 1 .
$$

If there is some $\lambda_{j}$ such that $K \lambda<\left|\lambda_{j}\right|$, then by (4.9), we see that, for any $x \in \mathbb{R}^{n}$,

$$
\left|B_{j}\right| \omega\left(x, \frac{\left|\lambda_{j}\right|}{\lambda\left|B_{j}\right| \sup _{x \in B_{j}} \rho\left(x,\left|B_{j}\right|\right)}\right)>\left|B_{j}\right| \omega\left[x, K \inf _{x \in B_{j}} \omega^{-1}\left(x,\left|B_{j}\right|^{-1}\right)\right] \geq 1
$$


which contradicts (4.11). Hence $K \lambda \geq\left|\lambda_{j}\right|$ for all $\lambda_{j}$. Since $\omega$ is of uniformly upper-type 1 , we deduce that

$$
\begin{aligned}
\left|B_{j}\right| \omega\left(x, \frac{\left|\lambda_{j}\right|}{\lambda\left|B_{j}\right| \sup _{x \in B_{j}} \rho\left(x,\left|B_{j}\right|\right)}\right) & \geq \frac{\left|\lambda_{i}\right|}{K \lambda}\left|B_{j}\right| \omega\left[x, K \inf _{x \in B_{j}} \omega^{-1}\left(x,\left|B_{j}\right|^{-1}\right)\right] \\
& \geq \frac{\left|\lambda_{i}\right|}{K \lambda},
\end{aligned}
$$

which, together with (4.11) and the definition of $\Lambda\left(\left\{\lambda_{j} a_{j}\right\}_{j}\right)$ in Theorem 3.1, completes the proof of Lemma 4.4 .

We are now ready to prove Theorem 4.1.

Proof of Theorem 4.1. Let $g \in \mathrm{BMO}_{\rho, L^{*}}^{M}\left(\mathbb{R}^{n}\right)$. For any $f \in H_{\omega, \text { fin }}^{2, \widetilde{M}}\left(\mathbb{R}^{n}\right) \subset$ $H_{\omega, L}\left(\mathbb{R}^{n}\right)$, we have $f \in L^{2}\left(\mathbb{R}^{n}\right)$ and hence, $t^{2} L e^{-t^{2} L} f \in\left(T_{\omega}\left(\mathbb{R}_{+}^{n+1}\right) \cap\right.$ $\left.T_{2}^{2}\left(\mathbb{R}_{+}^{n+1}\right)\right)$ by Lemma 2.4. By Theorem 3.1, there exist $\left\{\lambda_{j}\right\}_{j=1}^{\infty} \subset \mathbb{C}$ and $(\omega, \infty)$-atoms $\left\{a_{j}\right\}_{j=1}^{\infty}$ supported in $\left\{\widehat{B}_{j}\right\}_{j=1}^{\infty}$ such that (3.2) holds. Note that $g$ satisfies (4.8) with $q=2$ (by Lemma 4.1), which, together with Lemmas 4.2 and 4.3, the Hölder inequality, and Lemma 4.4(iii), yields

$$
\begin{aligned}
|\langle g, f\rangle| & =\left|C_{\widetilde{M}} \iint_{\mathbb{R}_{+}^{n+1}}\left(t^{2} L^{*}\right)^{M} e^{-t^{2} L^{*}} g(x) \overline{t^{2} L e^{-t^{2} L} f(x)} \frac{d x d t}{t}\right| \\
& \lesssim \sum_{j=1}^{\infty}\left|\lambda_{j}\right| \iint_{\mathbb{R}_{+}^{n+1}}\left|\left(t^{2} L^{*}\right)^{M} e^{-t^{2} L^{*}} g(x) \overline{a_{j}(x, t)}\right| \frac{d x d t}{t} \\
& \lesssim \sum_{j=1}^{\infty}\left|\lambda_{j}\right|\left\|a_{j}\right\|_{T_{2}^{2}\left(\mathbb{R}_{+}^{n+1}\right)}\left(\iint_{\widehat{B}_{j}}\left|\left(t^{2} L^{*}\right)^{M} e^{-t^{2} L^{*}} g(x)\right|^{2} \frac{d x d t}{t}\right)^{1 / 2} \\
& \lesssim \sum_{j=1}^{\infty}\left|\lambda_{j}\right|\|g\|_{\mathrm{BMO}_{\rho, L^{*}}^{M}\left(\mathbb{R}^{n}\right)} \lesssim\left\|t^{2} L e^{-t^{2} L} f\right\|_{T_{\omega}\left(\mathbb{R}_{+}^{n+1}\right)}\|g\|_{\mathrm{BMO}_{\rho, L^{*}}^{M}\left(\mathbb{R}^{n}\right)} \\
& \sim\|f\|_{H_{\omega, L}\left(\mathbb{R}^{n}\right)}\|g\|_{\mathrm{BMO}_{\rho, L^{*}}^{M}\left(\mathbb{R}^{n}\right)} .
\end{aligned}
$$

Then by a density argument via Corollary 4.2, we obtain (i).

Conversely, let $\ell \in\left(H_{\omega, L}\left(\mathbb{R}^{n}\right)\right)^{*}$. For any $(\omega, 2, M, \epsilon)$-molecule $\alpha$, it follows from 4.3 that $\|\alpha\|_{H_{\omega, L}\left(\mathbb{R}^{n}\right)} \lesssim 1$. Thus $|\ell(\alpha)| \lesssim\|\ell\|_{\left(H_{\omega, L}\left(\mathbb{R}^{n}\right)\right)^{*}}$, which implies that $\ell \in \mathcal{M}_{\omega, L^{*}}^{M}\left(\mathbb{R}^{n}\right)$.

To finish the proof of (ii), we still need to show that $\ell \in \mathrm{BMO}_{\rho, L^{*}}^{M}\left(\mathbb{R}^{n}\right)$. To this end, for any ball $B$, let $\phi \in L^{2}(B)$ with $\|\phi\|_{L^{2}(B)} \leq \frac{1}{|B|^{1 / 2} \sup _{x \in B} \rho(x,|B|)}$ 
and $\widetilde{\alpha} \equiv\left(I-\left[I+r_{B}^{2} L\right]^{-1}\right)^{M} \phi$. Then from Lemma 2.3, we deduce that, for each $j \in \mathbb{Z}_{+}$and each $k=0,1, \ldots, M$,

$$
\begin{aligned}
\left\|\left(r_{B}^{2} L\right)^{-k} \widetilde{\alpha}\right\|_{L^{2}\left(U_{j}(B)\right)} & =\left\|\left(I-\left[I+r_{B}^{2} L\right]^{-1}\right)^{M-k}\left(I+r_{B}^{2} L\right)^{-k} \phi\right\|_{L^{2}\left(U_{j}(B)\right)} \\
& \lesssim \exp \left\{-\frac{\operatorname{dist}\left(B, U_{j}(B)\right)}{c r_{B}}\right\}\|\phi\|_{L^{2}(B)} \\
& \lesssim 2^{-2 j(M+\epsilon)} 2^{j n\left(\frac{1}{p_{\omega}}-\frac{1}{2}\right)} \frac{1}{\left|2^{j} B\right|^{\frac{1}{2}} \sup _{x \in B} \rho\left(x,\left|2^{j} B\right|\right)} \\
& \lesssim 2^{-2 j \epsilon} \frac{1}{\left|2^{j} B\right|^{\frac{1}{2}} \sup _{x \in B} \rho\left(x,\left|2^{j} B\right|\right)},
\end{aligned}
$$

where $c$ is as in Lemma 2.3 and $2 M>n\left(\frac{1}{p_{\omega}}-\frac{1}{2}\right)$. Thus $\widetilde{\alpha}$ is a multiple of an $(\omega, 2, M, \epsilon)$-molecule. Since $\left(I-\left(\left[I+t^{2} L\right]^{-1}\right)^{*}\right)^{M} \ell$ is well defined and belongs to $L_{\text {loc }}^{2}\left(\mathbb{R}^{n}\right)$ for any fixed $t>0$, we have

$$
\begin{aligned}
\left|\left\langle\left(I-\left[\left(I+r_{B}^{2} L\right)^{-1}\right]^{*}\right)^{M} \ell, \phi\right\rangle\right| & =\left|\left\langle\ell,\left(I-\left[I+r_{B}^{2} L\right]^{-1}\right)^{M} \phi\right\rangle\right| \\
& =|\langle\ell, \widetilde{\alpha}\rangle| \lesssim\|\ell\|_{\left(H_{\omega, L}\left(\mathbb{R}^{n}\right)\right)^{*}}
\end{aligned}
$$

which further implies that

$$
\begin{aligned}
& \frac{1}{\sup _{x \in B} \rho(x,|B|)}\left(\frac{1}{|B|} \int_{B}\left|\left(I-\left[\left(I+r_{B}^{2} L\right)^{-1}\right]^{*}\right)^{M} \ell(x)\right|^{2} d x\right)^{1 / 2} \\
& =\sup _{\|\phi\|_{L^{2}(B)} \leq 1}\left|\left\langle\ell,\left(I-\left[I+r_{B}^{2} L\right]^{-1}\right)^{M} \frac{\phi}{|B|^{1 / 2} \sup _{x \in B} \rho(x,|B|)}\right\rangle\right| \\
& \lesssim\|\ell\|_{\left(H_{\omega, L}\left(\mathbb{R}^{n}\right)\right)^{*} .}
\end{aligned}
$$

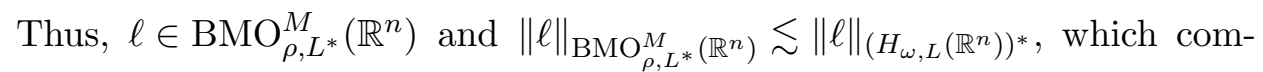
pletes the proof of Theorem 4.1.

REMARK 4.2. It follows from Theorem 4.1 that the spaces $\mathrm{BMO}_{\rho, L}^{M}\left(\mathbb{R}^{n}\right)$ for all $M>\frac{n}{2}\left(\frac{1}{p_{\omega}}-\frac{1}{2}\right)$ coincide with equivalent norms. Thus, in what follows, we denote $\mathrm{BMO}_{\rho, L}^{M}\left(\mathbb{R}^{n}\right)$ simply by $\mathrm{BMO}_{\rho, L}\left(\mathbb{R}^{n}\right)$.

\subsection{The Carleson measure and the John-Nirenberg inequality}

In this section, we characterize the space $\mathrm{BMO}_{\rho, L^{*}}\left(\mathbb{R}^{n}\right)$ via the $\rho$-Carleson measure and establish the John-Nirenberg inequality for elements in $\mathrm{BMO}_{\rho, L^{*}}\left(\mathbb{R}^{n}\right)$, where $L^{*}$ denotes the conjugate operator of $L$ in $L^{2}\left(\mathbb{R}^{n}\right)$. 
We call a measure $d \mu$ on $\mathbb{R}_{+}^{n+1}$ a $\rho$-Carleson measure if

$$
\|d \mu\|_{\rho} \equiv \sup _{B \subset \mathbb{R}^{n}}\left\{\frac{1}{|B|\left[\sup _{x \in B} \rho(x,|B|)\right]^{2}} \iint_{\widehat{B}}|d \mu|\right\}^{1 / 2}<\infty
$$

where the supremum is taken over all balls $B$ of $\mathbb{R}^{n}$ and where $\widehat{B}$ denotes the tent over $B$.

THEOREM 4.2. Let $\omega$ satisfy Assumption (C), and let $M>\frac{n}{2}\left(\frac{1}{p_{\omega}}-\frac{1}{2}\right)$.

(i) If $f \in \mathrm{BMO}_{\rho, L^{*}}\left(\mathbb{R}^{n}\right)$, then $d \mu_{f}$ is a $\rho$-Carleson measure and there exists a positive constant $C$ independent of $f$ such that $\left\|d \mu_{f}\right\|_{\rho} \leq$ $C\|f\|_{\mathrm{BMO}_{\rho, L^{*}}\left(\mathbb{R}^{n}\right)}^{2}$, where

$$
d \mu_{f} \equiv\left|\left(t^{2} L^{*}\right)^{M} e^{-t^{2} L^{*}} f(x)\right|^{2} \frac{d x d t}{t} .
$$

(ii) Conversely, if $f \in \mathcal{M}_{\omega, L^{*}}^{M}\left(\mathbb{R}^{n}\right)$ satisfies (4.8) with certain $q \in\left(p_{L^{*}}, 2\right]$ and $\epsilon_{1}>0$, (4.9) holds, and $d \mu_{f}$ is a $\rho$-Carleson measure, then $f \in$ $\mathrm{BMO}_{\rho, L^{*}}\left(\mathbb{R}^{n}\right)$ and there exists a positive constant $C$ independent of $f$ such that $\|f\|_{\mathrm{BMO}_{\rho, L^{*}}\left(\mathbb{R}^{n}\right)}^{2} \leq C\left\|d \mu_{f}\right\|_{\rho}$, where $d \mu_{f}$ is as in (4.13).

Proof. It follows from Lemma 4.2 that (i) holds. have

To show (ii), let $\widetilde{M}>M+\epsilon_{1}+\frac{n}{4}$ and $\epsilon>n\left(\frac{1}{p_{\omega}}-\frac{1}{p_{\omega}^{+}}\right)$. By Lemma 4.3, we

$$
\langle f, g\rangle=\widetilde{C}_{M} \iint_{\mathbb{R}_{+}^{n+1}}\left(t^{2} L^{*}\right)^{M} e^{-t^{2} L^{*}} f(x) \overline{t^{2} L e^{-t^{2} L} g(x)} \frac{d x d t}{t},
$$

where $g$ is a finite combination of $\left(\omega, q^{\prime}, \widetilde{M}, \epsilon\right)$-molecules and $q^{\prime}=\frac{q}{q-1}$. Then by (4.12), we obtain that

$$
|\langle f, g\rangle| \lesssim\left\|d \mu_{f}\right\|_{\rho}\|g\|_{H_{\omega, L}\left(\mathbb{R}^{n}\right)} .
$$

Since $H_{\omega, \text { fin }}^{q^{\prime}, \widetilde{M}}$ is dense in $H_{\omega, L}\left(\mathbb{R}^{n}\right)$, we then obtain $f \in\left(H_{\omega, L}\left(\mathbb{R}^{n}\right)\right)^{*}$, which combined with Theorem 4.1 implies that $f \in \mathrm{BMO}_{\rho, L^{*}}\left(\mathbb{R}^{n}\right)$ and $\|f\|_{\mathrm{BMO}_{\rho, L^{*}}\left(\mathbb{R}^{n}\right)} \lesssim\left\|d u_{f}\right\|_{\rho}$. This finishes the proof of Theorem 4.2.

By the same arguments as in the proof of [24, Theorem 6.2], we obtain the following result.

THEOREM 4.3. Let $\omega$ satisfy Assumption (C), let (4.9) hold, and let $M>$ $\frac{n}{2}\left(\frac{1}{p_{\omega}}-\frac{1}{2}\right)$. Then the spaces $\mathrm{BMO}_{\rho, L^{*}}^{q, M}\left(\mathbb{R}^{n}\right)$ for all $q \in\left(p_{L^{*}}, \widetilde{p}_{L^{*}}\right)$ coincide with equivalent norms. 
It could be of interest to put forward the following comment: these kinds of results are a well-known consequence of John-Nirenberg inequalities, as explained in this section. Recently, such self-improving properties have been studied in a very abstract setting (see [9], [27], [7], [11]). Moreover, in [9], applications for functional spaces (Hardy spaces and Sobolev spaces) associated to the same (than here) second-order divergence operator are obtained. In [9], [27], and [7], the main assumption to get this self-improving property (the John-Nirenberg inequality) is related to the behavior of the "weight" $\rho$, if it is doubling or increasing (with respect to the ball). They only consider weights, which are " $x$ "-independent. So the results obtained in the present article are interesting since they deal with an " $x$ "-dependent weight $\rho$. However, whether it is possible to compare Assumption (C) and (4.9) (required in Theorem 4.3) with the doubling property required for an " $x$ "-independent weight is still an interesting question. We believe that they are in general incomparable.

\section{$\S 5$. Some applications}

In this section, we establish the boundedness on Musielak-Orlicz Hardy spaces of the Riesz transform and the Littlewood-Paley $g$-function associated with the operator $L$ as in (1.2).

Recall that the Littlewood-Paley $g$-function $g_{L}$ is defined by setting, for all $f \in L^{2}\left(\mathbb{R}^{n}\right)$ and all $x \in \mathbb{R}^{n}$,

$$
g_{L} f(x) \equiv\left(\int_{0}^{\infty}\left|t^{2} L e^{-t^{2} L} f(x)\right|^{2} \frac{d t}{t}\right)^{1 / 2}
$$

By [22, Proof of Theorem 3.4], we know that $g_{L}$ is bounded on $L^{2}\left(\mathbb{R}^{n}\right)$.

Analogously to [22, Theorems 3.2, 3.4] and [24, Theorem 7.1], we have the following result.

Theorem 5.1. Let $\omega$ satisfy Assumption $(C)$, and let $p \in\left(p_{L}, 2\right]$. Suppose that the nonnegative sublinear operator or linear operator $T$ is bounded on $L^{p}\left(\mathbb{R}^{n}\right)$ and that there exist $C>0, M \in \mathbb{N}$, and $M>\frac{n}{2}\left(\frac{1}{p_{\omega}}-\frac{1}{2}\right)$ such that, for all closed sets $E, F$ in $\mathbb{R}^{n}$ with $\operatorname{dist}(E, F)>0$ and for all $f \in L^{p}\left(\mathbb{R}^{n}\right)$ supported in $E$,

$$
\left\|T\left(I-e^{-t L}\right)^{M} f\right\|_{L^{p}(F)} \leq C\left(\frac{t}{\operatorname{dist}(E, F)^{2}}\right)^{M}\|f\|_{L^{p}(E)}
$$


and

$$
\left\|T\left(t L e^{-t L}\right)^{M} f\right\|_{L^{p}(F)} \leq C\left(\frac{t}{\operatorname{dist}(E, F)^{2}}\right)^{M}\|f\|_{L^{p}(E)}
$$

for all $t>0$. Then $T$ extends to a bounded sublinear or linear operator from $H_{\omega, L}\left(\mathbb{R}^{n}\right)$ to $L(\omega)$. In particular, the Riesz transform $\nabla L^{-1 / 2}$ and the Littlewood-Paley $g$-function $g_{L}$ are bounded from $H_{\omega, L}\left(\mathbb{R}^{n}\right)$ to $L(\omega)$.

Proof. Before coming to the proof, we need the following useful result, which is a slight variant of [24, Lemma 5.1] on the boundedness of linear or nonnegative sublinear operators from $H_{\omega, L}\left(\mathbb{R}^{n}\right)$ to $L(\omega)$.

Lemma 5.1. Let $q \in\left(p_{L}, 2\right]$, let $\omega$ satisfy Assumption $(C)$, let $M>$ $\frac{n}{2}\left(\frac{1}{p_{\omega}}-\frac{1}{2}\right)$, and let $\epsilon>n\left(\frac{1}{p_{\omega}}-\frac{1}{p_{\omega}^{+}}\right)$. Suppose that $T$ is a nonnegative sublinear (resp., linear) operator which maps $L^{q}\left(\mathbb{R}^{n}\right)$ continuously into weak- $L^{q}\left(\mathbb{R}^{n}\right)$. If there exists a positive constant $C$ such that, for all $(\omega, \infty, M, \epsilon)$-molecules $\alpha$ adapted to balls $B$ and for $\lambda \in \mathbb{C}$,

$$
\int_{\mathbb{R}^{n}} \omega(x, T(\lambda \alpha)(x)) d x \leq C|B| \inf _{x \in \mathbb{R}^{n}} \omega\left(x, \frac{|\lambda|}{|B| \sup _{x \in B} \rho(x,|B|)}\right),
$$

then $T$ extends to a bounded sublinear (resp., linear) operator from $H_{\omega, L}\left(\mathbb{R}^{n}\right)$ to $L(\omega)$; moreover, there exists a positive constant $\widetilde{C}$ such that for all $f \in$ $H_{\omega, L}\left(\mathbb{R}^{n}\right)$, we have $\|T f\|_{L(\omega)} \leq \widetilde{C}\|f\|_{H_{\omega, L}\left(\mathbb{R}^{n}\right)}$.

Proof of Lemma 5.1. It follows from Proposition 4.2 that for every $f \in$ $H_{\omega, L}\left(\mathbb{R}^{n}\right) \cap L^{2}\left(\mathbb{R}^{n}\right)$, we have $f \in L^{q}\left(\mathbb{R}^{n}\right)$ with $q \in\left(p_{L}, 2\right]$, and there exists $\left\{\lambda_{j}\right\}_{j=1}^{\infty} \subset \mathbb{C}$ and $(\omega, \infty, M, \epsilon)$-molecules $\left\{\alpha_{j}\right\}_{j=1}^{\infty}$ such that $f=\sum_{j=1}^{\infty} \lambda_{j} \alpha_{j}$ in both $H_{\omega, L}\left(\mathbb{R}^{n}\right)$ and $L^{q}\left(\mathbb{R}^{n}\right)$. Moreover, $\Lambda\left(\left\{\lambda_{j} \alpha_{j}\right\}_{j}\right) \lesssim\|f\|_{H_{\omega, L}\left(\mathbb{R}^{n}\right)}$. Thus if $T$ is linear, then it follows from the fact that $T$ is of weak type $(q, q)$ that $T(f)=\sum_{j=1}^{\infty} T\left(\lambda_{j} \alpha_{j}\right)$ almost everywhere.

If $T$ is a nonnegative sublinear operator, then

$$
\begin{aligned}
& \sup _{t>0} t^{\frac{1}{q}}\left|\left\{x \in \mathbb{R}^{n}:\left|T(f)(x)-T\left(\sum_{j=1}^{N} \lambda_{j} \alpha_{j}\right)(x)\right|>t\right\}\right| \\
& \lesssim\left\|f-\sum_{j=1}^{N} \lambda_{j} \alpha_{j}\right\|_{L^{q}\left(\mathbb{R}^{n}\right)} \rightarrow 0
\end{aligned}
$$


as $N \rightarrow \infty$. Thus there exists a subsequence $\left\{N_{k}\right\}_{k} \subset \mathbb{N}$ such that

$$
T\left(\sum_{j=1}^{N_{k}} \lambda_{j} \alpha_{j}\right) \rightarrow T(f)
$$

almost everywhere, as $k \rightarrow \infty$, which together with the nonnegativity and the sublinearity of $T$ further implies that

$$
\begin{aligned}
& T(f)-\sum_{j=1}^{\infty} T\left(\lambda_{j} \alpha_{j}\right) \\
& \quad=T(f)-T\left(\sum_{j=1}^{N_{k}} \lambda_{j} \alpha_{j}\right)+T\left(\sum_{j=1}^{N_{k}} \lambda_{j} \alpha_{j}\right)-\sum_{j=1}^{\infty} T\left(\lambda_{j} \alpha_{j}\right) \\
& \quad \leq T(f)-T\left(\sum_{j=1}^{N_{k}} \lambda_{j} \alpha_{j}\right) .
\end{aligned}
$$

By letting $k \rightarrow \infty$, we see that $T(f) \leq \sum_{j=1}^{\infty} T\left(\lambda_{j} \alpha_{j}\right)$ almost everywhere. Thus by the subadditivity and the continuity of $\omega$ and (5.3), we finally obtain

$$
\begin{aligned}
\int_{\mathbb{R}^{n}} \omega(x, T(f)(x)) d x & \lesssim \sum_{j=1}^{\infty} \int_{\mathbb{R}^{n}} \omega\left(x, T\left(\lambda_{j} \alpha_{j}\right)(x)\right) d x \\
& \lesssim \sum_{j=1}^{\infty}\left|B_{j}\right| \inf _{x \in \mathbb{R}^{n}} \omega\left(x, \frac{\left|\lambda_{j}\right|}{\left|B_{j}\right| \sup _{x \in B_{j}} \rho\left(x,\left|B_{j}\right|\right)}\right)
\end{aligned}
$$

which implies that $\|T(f)\|_{L(\omega)} \lesssim \Lambda\left(\left\{\lambda_{j} \alpha_{j}\right\}_{j}\right) \lesssim\|f\|_{H_{\omega, L}\left(\mathbb{R}^{n}\right)}$. This, combined with the density of $H_{\omega, L}\left(\mathbb{R}^{n}\right) \cap L^{2}\left(\mathbb{R}^{n}\right)$ in $H_{\omega, L}\left(\mathbb{R}^{n}\right)$, then completes the proof of Lemma 5.1.

We are now in position to prove Theorem 5.1.

Proof of Theorem 5.1. Let $\epsilon>n\left(\frac{1}{p_{\omega}}-\frac{1}{\widetilde{p}_{\omega}}\right)$, where $\widetilde{p}_{\omega}$ is as in Convention (B). Since $T$ is bounded on $L^{p}\left(\mathbb{R}^{n}\right)$, by Lemma 5.1, to show that $T$ is bounded from $H_{\omega, L}\left(\mathbb{R}^{n}\right)$ to $L(\omega)$ it suffices to show that, for all $\lambda \in \mathbb{C}$ and for $(\omega, \infty, M, \epsilon)$-molecules $\alpha$ adapted to balls $B$,

$$
\int_{\mathbb{R}^{n}} \omega(x, T(\lambda \alpha)(x)) d x \lesssim|B| \inf _{x \in \mathbb{R}^{n}} \omega\left(x, \frac{|\lambda|}{|B| \sup _{x \in B} \rho(x,|B|)}\right) .
$$


To prove (5.4), we write

$$
\begin{aligned}
\int_{\mathbb{R}^{n}} \omega & (x, T(\lambda \alpha)(x)) d x \\
\leq & \int_{\mathbb{R}^{n}} \omega\left(x,|\lambda| T\left(\left[I-e^{-r_{B}^{2} L}\right]^{M} \alpha\right)(x)\right) d x \\
& +\int_{\mathbb{R}^{n}} \omega\left(x,|\lambda| T\left(\left(I-\left[I-e^{-r_{B}^{2} L}\right]^{M}\right) \alpha\right)(x)\right) d x \\
\lesssim & \sum_{j=0}^{\infty} \int_{\mathbb{R}^{n}} \omega\left(x,|\lambda| T\left(\left[I-e^{-r_{B}^{2} L}\right]^{M}\left(\alpha \chi_{U_{j}(B)}\right)\right)(x)\right) d x \\
& +\sum_{j=0}^{\infty} \sup _{1 \leq k \leq M} \int_{\mathbb{R}^{n}} \omega\left(x,|\lambda| T\left\{\left[\frac{k}{M} r_{B}^{2} L e^{-\frac{k}{M} r_{B}^{2} L}\right]^{M}\right.\right. \\
& \left.\left.\times\left(\chi_{U_{j}(B)}\left(r_{B}^{-2} L^{-1}\right)^{M} \alpha\right)\right\}(x)\right) d x \\
\equiv & \sum_{j=0}^{\infty} \mathrm{H}_{j}+\sum_{j=0}^{\infty} \mathrm{I}_{j} .
\end{aligned}
$$

For each $j \geq 0$, let $B_{j} \equiv 2^{j} B$. By the Hölder inequality, we obtain

$$
\begin{aligned}
\mathrm{H}_{j} & \lesssim \sum_{k=0}^{\infty} \int_{U_{k}\left(B_{j}\right)} \omega\left(x,|\lambda| T\left(\left[I-e^{-r_{B}^{2} L}\right]^{M}\left(\alpha \chi_{U_{j}(B)}\right)\right)(x)\right) d x \\
& \lesssim \sum_{k=0}^{\infty} \int_{2^{k} B_{j}} \omega\left(x,|\lambda| \chi_{U_{k}\left(B_{j}\right)}(x) T\left(\left[I-e^{-r_{B}^{2} L}\right]^{M}\left(\alpha \chi_{U_{j}(B)}\right)\right)(x)\right) d x \\
& \lesssim \sum_{k=0}^{\infty}\left|2^{k} B_{j}\right| \inf _{x \in \mathbb{R}^{n}} \omega\left(x, \frac{|\lambda|}{\left|2^{k} B_{j}\right|} \int_{U_{k}\left(B_{j}\right)} T\left(\left[I-e^{-r_{B}^{2} L}\right]^{M}\left(\alpha \chi_{U_{j}(B)}\right)\right)(x) d x\right) \\
& \lesssim \sum_{k=0}^{\infty}\left|2^{k} B_{j}\right| \inf _{x \in \mathbb{R}^{n}} \omega\left(x, \frac{|\lambda|}{\left|2^{k} B_{j}\right|^{1 / p}}\left\|T\left(\left[I-e^{-r_{B}^{2} L}\right]^{M}\left(\alpha \chi_{U_{j}(B)}\right)\right)\right\|_{L^{p}\left(U_{k}\left(B_{j}\right)\right)}\right) .
\end{aligned}
$$

By the $L^{p}\left(\mathbb{R}^{n}\right)$-boundedness of $T$, Lemma 2.3, and (5.1), we have for $k=$ $0,1,2$,

$$
\left\|T\left(\left[I-e^{-r_{B}^{2} L}\right]^{M}\left(\alpha \chi_{U_{j}(B)}\right)\right)\right\|_{L^{p}\left(U_{k}\left(B_{j}\right)\right)} \lesssim\|\alpha\|_{L^{p}\left(U_{j}(B)\right)},
$$

and that for $k \geq 3$,

$$
\left\|T\left(\left[I-e^{-r_{B}^{2} L}\right]^{M}\left(\alpha \chi_{U_{j}(B)}\right)\right)\right\|_{L^{p}\left(U_{k}\left(B_{j}\right)\right)} \lesssim\left(\frac{1}{2^{k+j}}\right)^{2 M}\|\alpha\|_{L^{p}\left(U_{j}(B)\right)}^{2},
$$


which, together with Definition 4.2 and $2 M p_{\omega}>n\left(1-\frac{p_{\omega}}{2}\right)$, implies that

$$
\begin{aligned}
& \mathrm{H}_{j} \lesssim\left|B_{j}\right| \inf _{x \in \mathbb{R}^{n}} \omega\left(x, \frac{|\lambda| 2^{-j \epsilon}}{\left|B_{j}\right| \sup _{x \in B_{j}} \rho\left(x,\left|B_{j}\right|\right)}\right) \\
& +\sum_{k=3}^{\infty}\left|2^{k} B_{j}\right| \inf _{x \in \mathbb{R}^{n}} \omega\left(x, \frac{|\lambda| 2^{-(2 M)(j+k)-j \epsilon}}{\left|2^{k} B_{j}\right|^{1 / p}\left|B_{j}\right|^{1-\frac{1}{p}} \sup _{x \in B_{j}} \rho\left(x,\left|B_{j}\right|\right)}\right) \\
& \lesssim 2^{-j p_{\omega} \epsilon}\left\{1+\sum_{k=3}^{\infty} 2^{k n\left(1-\frac{p_{\omega}}{p}\right)} 2^{-2 M p_{\omega}(j+k)}\right\}\left|B_{j}\right| \\
& \times \inf _{x \in \mathbb{R}^{n}} \omega\left(x, \frac{|\lambda|}{\left|B_{j}\right| \sup _{x \in B_{j}} \rho\left(x,\left|B_{j}\right|\right)}\right) \\
& \lesssim 2^{-j p_{\omega} \epsilon}\left|B_{j}\right| \inf _{x \in \mathbb{R}^{n}} \omega\left(x, \frac{|\lambda|}{\left|B_{j}\right| \sup _{x \in B_{j}} \rho\left(x,\left|B_{j}\right|\right)}\right) .
\end{aligned}
$$

Since $\omega^{-1}$ is of uniformly lower-type $\frac{1}{\tilde{p}_{\omega}}$ and $\epsilon>n\left(\frac{1}{p_{\omega}}-\frac{1}{\tilde{p}_{\omega}}\right)$, we further have

$$
\begin{aligned}
\sum_{j=0}^{\infty} \mathrm{H}_{j} \lesssim & \sum_{j=0}^{\infty} 2^{-j p_{\omega} \epsilon}\left|B_{j}\right|\left\{\frac{|B| \sup _{x \in B} \rho(x,|B|)}{\left|B_{j}\right| \sup _{x \in B_{j}} \rho\left(x,\left|B_{j}\right|\right)}\right\}^{p_{\omega}} \\
& \times \inf _{x \in \mathbb{R}^{n}} \omega\left(x, \frac{|\lambda|}{|B| \sup _{x \in B} \rho(x,|B|)}\right) \\
\lesssim & \sum_{j=0}^{\infty} 2^{-j p_{\omega} \epsilon}\left|B_{j}\right|\left\{\frac{|B|}{\left|B_{j}\right|}\right\}^{\frac{p_{\omega}}{\tilde{p}_{\omega}}} \inf _{x \in \mathbb{R}^{n}} \omega\left(x, \frac{|\lambda|}{|B| \sup _{x \in B} \rho(x,|B|)}\right) \\
\lesssim & \sum_{j=0}^{\infty} 2^{-j p_{\omega} \epsilon} 2^{j n\left(1-\frac{p_{\omega}}{\tilde{p}_{\omega}}\right)}|B| \inf _{x \in \mathbb{R}^{n}} \omega\left(x, \frac{|\lambda|}{|B| \sup _{x \in B} \rho(x,|B|)}\right) \\
\lesssim & |B| \inf _{x \in \mathbb{R}^{n}} \omega\left(x, \frac{|\lambda|}{|B| \sup _{x \in B} \rho(x,|B|)}\right) .
\end{aligned}
$$

Similarly, we have

$$
\sum_{j=0}^{\infty} \mathrm{I}_{j} \lesssim|B| \inf _{x \in \mathbb{R}^{n}} \omega\left(x, \frac{|\lambda|}{|B| \sup _{x \in B} \rho(x,|B|)}\right) .
$$

Thus, (5.4) holds, and hence, $T$ is bounded from $H_{\omega, L}\left(\mathbb{R}^{n}\right)$ to $L(\omega)$.

It was proved in [22, Theorem 3.4] that operators $g_{L}$ and $\nabla L^{-1 / 2}$ satisfy (5.1) and (5.2); thus $g_{L}$ and $\nabla L^{-1 / 2}$ are bounded from $H_{\omega, L}\left(\mathbb{R}^{n}\right)$ to $L(\omega)$, which finishes the proof of Theorem 5.1. 
Acknowledgments. This article developed out of the author's Ph.D. dissertation, and he would like to thank his thesis supervisor, Xuan Thinh Duong, for helpful comments and discussions. He also thanks the referees for their valuable suggestions and comments, which led to an improved presentation. The author's work was partially supported by an International Postgraduate Research Scholarship (IPRS) scholarship at Macquarie University, Australia.

\section{REFERENCES}

[1] P. Auscher, On necessary and sufficient conditions for $L^{p}$-estimates of Riesz transforms associated to elliptic operators on $\mathbb{R}^{n}$ and related estimates, Mem. Amer. Math. Soc. 186 (2007), no. 871. MR 2292385. DOI 10.1090/memo/0871.

[2] P. Auscher, X. T. Duong, and A. McIntosh, Boundedness of Banach space valued singular integral operators and Hardy spaces, in preparation.

[3] P. Auscher, S. Hofmann, M. Lacey, A. McIntosh, and P. Tchamitchian, The solution of the Kato square root problem for second order elliptic operators on $\mathbb{R}^{n}$, Ann. of Math. (2) 156 (2002), 633-654. MR 1933726. DOI 10.2307/3597201.

[4] P. Auscher, A. McIntosh, and E. Russ, Hardy spaces of differential forms on Riemannian manifolds, J. Geom. Anal. 18 (2008), 192-248. MR 2365673. DOI 10.1007/s12220-007-9003-x.

[5] P. Auscher and E. Russ, Hardy spaces and divergence operators on strongly Lipschitz domains of $\mathbb{R}^{n}$, J. Funct. Anal. 201 (2003), 148-184. MR 1986158. DOI 10.1016/S0022-1236(03)00059-4.

[6] P. Auscher and P. Tchamitchian, Square Root Problem for Divergence Operators and Related Topics, Astérisque 249, Soc. Math. France, Paris, 1998. MR 1651262.

[7] N. Badr, A. Jiménez del Toro, and J. M. Martell, $L^{p}$ self-improvement of generalized Poincaré inequalities in spaces of homogeneous type, J. Funct. Anal. 260 (2011), 3147-3188. MR 2776565. DOI 10.1016/j.jfa.2011.01.014.

[8] F. Bernicot, Use of Hardy spaces and interpolation, C. R. Math. Acad. Sci. Paris 346 (2008), 745-748. MR 2427074. DOI 10.1016/j.crma.2008.05.009.

[9] F. Bernicot and J. M. Martell, Self-improving properties for abstract Poincaré type inequalities, preprint, arXiv:1107.2260v1 [math.CA].

[10] F. Bernicot and J. Zhao, New abstract Hardy spaces, J. Funct. Anal. 255 (2008), 1761-1796. MR 2442082. DOI 10.1016/j.jfa.2008.06.018.

[11] - Abstract framework for John-Nirenberg inequalities and applications to Hardy spaces, Ann. Sc. Norm. Super. Pisa Cl. Sci. (5) 11 (2012), 475-501. MR 3059835.

[12] T. A. Bui, J. Cao, L. D. Ky, D. Yang, and S. Yang, Musielak-Orlicz-Hardy spaces associated with operators satisfying reinforced off-diagonal estimates, Anal. Geom. Metr. Spaces 1 (2012), 69-129. MR 3108869.

[13] R. R. Coifman, Y. Meyer, and E. M. Stein, Some new function spaces and their applications to harmonic analysis, J. Funct. Anal. 62 (1985), 304-335. MR 0791851. DOI 10.1016/0022-1236(85)90007-2.

[14] L. Diening, Maximal function on Musielak-Orlicz spaces and generalized Lebesgue spaces, Bull. Sci. Math. 129 (2005), 657-700. MR 2166733. DOI 10.1016/j.bulsci.2003.10.003. 
[15] X. T. Duong, J. Xiao, and L. Yan, Old and new Morrey spaces with heat kernel bounds, J. Fourier Anal. Appl. 13 (2007), 87-111. MR 2296729. DOI 10.1007/s00041-006-6057-2.

[16] X. T. Duong and L. Yan, Duality of Hardy and BMO spaces associated with operators with heat kernel bounds, J. Amer. Math. Soc. 18 (2005), 943-973. MR 2163867. DOI 10.1090/S0894-0347-05-00496-0.

[17] - New function spaces of BMO type, the John-Nirenberg inequality, interpolation, and applications, Comm. Pure Appl. Math. 58 (2005), 1375-1420. MR 2162784. DOI $10.1002 /$ cpa.20080.

[18] C. Fefferman and E. M. Stein, $H^{p}$ spaces of several variables, Acta Math. 129 (1972), 137-193. MR 0447953.

[19] E. Harboure, O. Salinas, and B. Viviani, A look at $\mathrm{BMO}_{\varphi}(\omega)$ through Carleson measures, J. Fourier Anal. Appl. 13 (2007), 267-284. MR 2334610. DOI 10.1007/s00041-005-5044-3.

[20] S. Hofmann, G. Lu, D. Mitrea, M. Mitrea, and L. Yan, Hardy spaces associated to non-negative self-adjoint operators satisfying Davies-Gaffney estimates, Mem. Amer. Math. Soc. 214 (2011), no. 1007. MR 2868142. DOI 10.1090/S0065-9266-2011-00624-6.

[21] S. Hofmann and J. M. Martell, $L^{p}$ bounds for Riesz transforms and square roots associated to second order elliptic operators, Publ. Mat. 47 (2003), 497-515. MR 2006497. DOI 10.5565/PUBLMAT_47203_12.

[22] S. Hofmann and S. Mayboroda, Hardy and BMO spaces associated to divergence form elliptic operators, Math. Ann. 344 (2009), 37-116. MR 2481054. DOI 10.1007/s00208-008-0295-3.

[23] S. Janson, Generalizations of Lipschitz spaces and an application to Hardy spaces and bounded mean oscillation, Duke Math. J. 47 (1980), 959-982. MR 0596123.

[24] R. Jiang and D. Yang, New Orlicz-Hardy spaces associated with divergence form elliptic operators, J. Funct. Anal. 258 (2010), 1167-1224. MR 2565837. DOI 10.1016/j.jfa.2009.10.018.

[25] — - Orlicz-Hardy spaces associated with operators satisfying Davies-Gaffney estimates, Commun. Contemp. Math. 13 (2011), 331-373. MR 2794490. DOI 10.1142/S0219199711004221.

[26] R. Jiang, D. Yang, and Y. Zhou, Orlicz-Hardy spaces associated with operators, Sci. China Ser. A 52 (2009), 1042-1080. MR 2505009. DOI 10.1007/s11425-008-0136-6.

[27] A. Jiménez del Toro and J. M. Martell, Self-improvement of Poincaré type inequalities associated with approximations of the identity and semigroups, Potential Anal. 38 (2013), 805-841. MR 3034601. DOI 10.1007/s11118-012-9298-5.

[28] L. D. Ky, New Hardy spaces of Musielak-Orlicz type and boundedness of sublinear operators, Integral Equations Operator Theory 78 (2014), 115-150. MR 3147406. DOI 10.1007/s00020-013-2111-z.

[29] Y. Liang, D. Yang, and S. Yang, Applications of Orlicz-Hardy spaces associated with operators satisfying Poisson estimates, Sci. China Math. 54 (2011), 2395-2426. MR 2859702. DOI 10.1007/s11425-011-4294-6.

[30] J. Musielak, Orlicz Spaces and Modular Spaces, Lecture Notes in Math. 1034, Springer, Berlin, 1983. MR 0724434.

[31] E. M. Stein, Harmonic Analysis: Real-Variable Methods, Orthogonality, and Oscillatory Integrals, Princeton Math. Ser. 43, Princeton University Press, Princeton, 1993. MR 1232192. 
[32] E. M. Stein and G. Weiss, On the theory of harmonic functions of several variables, I: The theory of $H^{p}$-spaces, Acta Math. 103 (1960), 25-62. MR 0121579.

[33] J.-O. Strömberg, Bounded mean oscillation with Orlicz norms and duality of Hardy spaces, Indiana Univ. Math. J. 28 (1979), 511-544. MR 0529683. DOI 10.1512/iumj.1979.28.28037.

[34] B. E. Viviani, An atomic decomposition of the predual of $\operatorname{BMO}(\rho)$, Rev. Mat. Iberoam. 3 (1987), 401-425. MR 0996824. DOI 10.4171/RMI/56.

[35] L. Yan, Classes of Hardy spaces associated with operators, duality theorem and applications, Trans. Amer. Math. Soc. 360 (2008), no. 8, 4383-4408. MR 2395177. DOI 10.1090/S0002-9947-08-04476-0.

[36] D. Yang and S. Yang, Musielak-Orlicz-Hardy spaces associated with operators and their applications, J. Geom. Anal. 24 (2014), 495-570. MR 3145932. DOI 10.1007/s12220012-9344-y.

[37] K. Yosida, Functional Analysis, 6th ed., Grundlehren Math. Wiss. 123, Springer, Berlin, 1980. MR 0617913.

Department of Mathematics

University of Pedagogy

Ho Chi Minh city

Vietnam

Current:

Department of Mathematics

Macquarie University

NSW 2109

Australia

tridung. tran@mq . edu . au

tridungsp@gmail.com 